Research Article: New Research / Cognition and Behavior

\title{
Opioid and sucrose craving are accompanied by unique behavioral and affective profiles after extended abstinence in male and female rats
}

https://doi.org/10.1523/ENEURO.0515-21.2022

Cite as: eNeuro 2022; 10.1523/ENEURO.0515-21.2022

Received: 14 December 2021

Revised: 17 February 2022

Accepted: 24 February 2022

This Early Release article has been peer-reviewed and accepted, but has not been through the composition and copyediting processes. The final version may differ slightly in style or formatting and will contain links to any extended data.

Alerts: Sign up at www.eneuro.org/alerts to receive customized email alerts when the fully formatted version of this article is published.

Copyright (? 2022 Mayberry et al.

This is an open-access article distributed under the terms of the Creative Commons Attribution 4.0 International license, which permits unrestricted use, distribution and reproduction in any medium provided that the original work is properly attributed. 


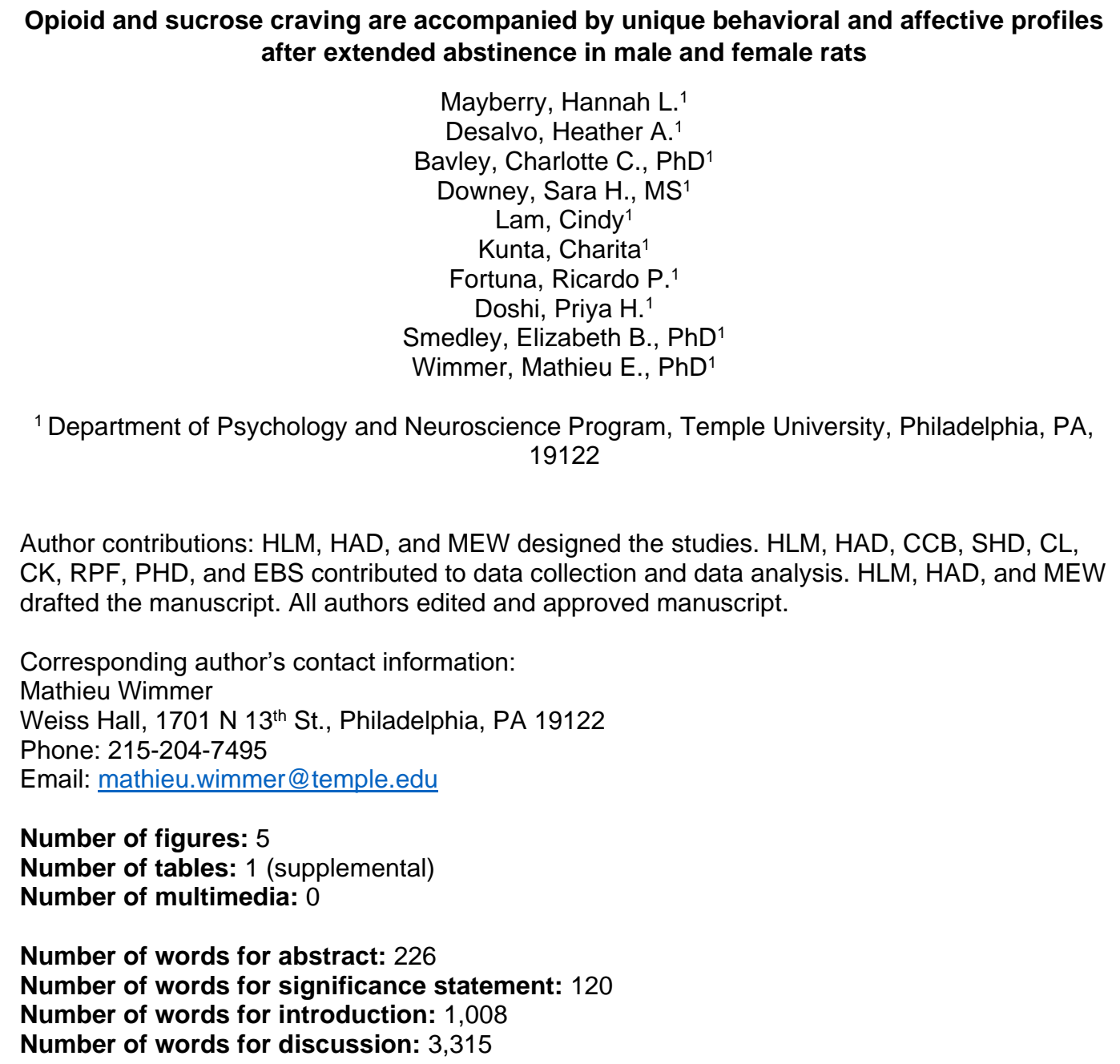

Acknowledgements: We thank the NIDA drug supply for gifting the heroin used in these studies. We would like to thank Carmen Dressler, Angela Bongiovanni, and Rachel Carpenter for their technical assistance.

Disclosures: Authors report no conflict of interest.

Funding: This work was supported by the National Institutes of Health grant NIH/NIDA DP1 DA046537, K01 DA039308 (MEW), and T32 DA007237 (CCB; Unterwald EM, PI). 


\section{Abstract}

Incubation of craving refers to the intensification of drug seeking behavior in response to reward-paired cues over the course of abstinence. In rodents, craving and drug-seeking behaviors have been measured by an increase in lever pressing in the absence of reinforcer availability in response to cue presentations. However, craving in rodents is difficult to define and little is known about the behavioral signatures that accompany increased drug-seeking behavior measured by lever pressing. The affective components of relapse are also important, but understudied in rodents. Hormonal fluctuations influence craving for psychostimulants, but little is known about the impact of the estrous cycle on opioid-seeking behavior. This study sought to delineate the behavioral and affective signatures associated with craving, and to examine the influence of the female estrous cycle on craving. Male and female rats underwent 10 days of intravenous opioid self-administration. Separate cohorts of control rats selfadministered oral sucrose, a natural non-drug reward. Cue-induced seeking tests were conducted after one or thirty days of forced abstinence. These sessions were recorded and scored for overall locomotion, instances of sniffing, grooming, or hyperactivity. Ultrasonic vocalizations were also recorded to determine affective profiles that accompany opioid seeking. Although active lever presses and overall locomotion increased unanimously over extended abstinence from heroin and sucrose, a sex and reinforcer-specific behavioral and affective signature of craving emerged. Furthermore, though the female estrous cycle did not affect taking or seeking, it appears to influence more granular behaviors.

\section{Significance statement}

Craving was recently added as a diagnostic criterion for substance use disorders. Positive and negative affect may contribute to relapse, though this domain is not fully understood in the context of cue-induced drug seeking in rodents. To enhance the definition of craving and to address potential sex differences, we recorded behaviors and ultrasonic vocalizations during early and late abstinence cue-induced relapse tests in male and female rats. The behavioral signatures associated with craving were sex- and reinforcer-specific. In females, the estrous cycle selectively influenced locomotion and hyperactivity during reinforcer seeking. These findings contribute to a better behavioral definition of craving, which lays the foundation to better understand the neural mechanisms underlying craving and drug seeking behaviors.

\section{Introduction}

Craving is an important hallmark of substance use disorders that was recently added as a diagnostic criterion in the DSM. Re-exposure to drug-paired cues often triggers craving, defined as a compulsion to use the drug. Craving intensifies for several weeks after drug use cessation in humans (Gawin and Kleber, 1986; O'Brien et al., 1992) and rodent models of addiction, referred to as "incubation of craving" (Grimm et al., 2001; Pickens et al., 2011). Incubation of craving is a beneficial and translationally relevant model for studying relapse because 1) it occurs in both humans and rodents, and 2) in rodents, it mirrors the condition in 
which drug use is discontinued and then individuals reintegrate into a familiar environment after some time away (hospitalization, incarceration, in-patient rehabilitation, etc.). Drug-related stimuli can gain salience over time and induce sensitization, known as incentive sensitization (Berridge and Robinson, 2016). One prominent construct in the addiction field is that incentive sensitization contributes to chronic, compulsive drug seeking, as well as other documented stereotyped behaviors during cue re-exposure (Tindell et al., 2005). This form of reward-related plasticity can last years after cessation of drug use (Paulson et al., 1991; Carr et al., 2020). Thus, incentive sensitization, which posits that drug-paired cues gain salience, likely contributes to incubation of craving where cue re-exposure elicits drug seeking even after prolonged abstinence. Importantly, these phenomena are thought to lead to high relapse rates. Drug craving is subjective in humans and difficult to model in rodents. In preclinical selfadministration paradigms, craving is often measured using the number of responses on a previously reward-associated lever when the reinforcer is not available. However, lever pressing does not capture other potentially important and more nuanced behaviors associated with drug seeking. This study built upon a wealth of previous behavioral sensitization studies and incorporated some of the drug-induced stereotypical behaviors in establishing a behavioral signature of opioid craving. Behavioral sensitization refers to documented, drug-induced, predictable (stereotyped) responses that emerge in both humans (Sax and Strakowski, 2001) and rodents (Steketee and Kalivas, 2011), and is not to be confused with incentive sensitization - a theoretical concept that stimuli can induce drug-seeking behavior. These specific stereotyped movements are predictive of drug-induced behavioral and neural plasticity and constitute the foundation of the behavioral signature defined in this study.

Craving for the natural reward sucrose also increases over extended abstinence and was used as a comparison to tease out opioid-specific profiles that accompany cue-induced reinforcer seeking. Sucrose is an important control group because it acts on many of the same neural pathways as drugs of abuse, and yet it is not the intended target of treatments aimed to 
reduce craving. Given that incubation of craving happens for many drugs of abuse as well as non-drug reinforcers like sucrose, this indicates that, in general, the brain is intensifying the cuereinforcer association over extended abstinence, regardless of reinforcer. Changes that are consistent across reinforcers suggest overlap in the pathways that drive this process, some of which can be adaptive (as is the case for remembering cues that are predictive of positive natural rewards). However, by isolating drug-specific changes, it is possible to disentangle the pathways that specifically drive maladaptive drug-cue association intensification throughout abstinence. These are promising targets for treating opioid craving without affecting the natural reward system.

Positive and negative affective states are thought to contribute to relapse potential (McKay et al., 1996; Rubin et al., 1996; Witkiewitz and Bowen, 2010; Becker and Koob, 2016) and clinical models of relapse include affective state (Witkiewitz and Marlatt, 2004). However, this domain is not often considered in preclinical models of reinstatement or cue-induced incubation of craving, particularly for opioids. Rodent affective states can be measured using ultrasonic vocalizations (USVs). Low-frequency 22-kHz USVs (18-33 kHz) correspond to negative-valence affective states and are emitted in situations of fear or anxiety. High-frequency $50-\mathrm{kHz}$ USVs $(38-90 \mathrm{kHz})$ correspond to positive-valence affective states and are emitted in response to rewarding situations such as social play and drug or food self-administration (Barker et al., 2015). Ultimately, USVs can elucidate affective components of opioid craving in rats.

There are no sex differences in incubation of heroin craving, as measured by active lever presses (Venniro et al., 2019); however, it is possible that males and females differ in terms of more granular addiction-related behaviors. For example, females show enhanced locomotor sensitization in response to experimenter-delivered methamphetamine (Robinson, 1984) and cocaine (Carr et al., 2020). Females also show greater incentive sensitization following intermittent access cocaine self-administration (Kawa and Robinson, 2019a). Limited 
studies have examined the role of biological sex in USV production. Female High Alcohol Drinking (HAD-1) rats emit more 50-kHz USVs during ethanol access compared to males (Mittal et al., 2019). It is unknown whether there is a sex-specific behavioral or affective signature associated with sucrose or opioid craving that persists into protracted abstinence.

Another important consideration that may influence some of these behaviors is the female estrous cycle. The estrous cycle affects addiction-like behaviors including cocaine CPP, and incubation of cocaine craving (Paris et al., 2014; Calipari et al., 2017; Nicolas et al., 2019). However, results related to whether the estrous cycle impacts opioid-related behaviors are conflicting. Furthermore, few studies have examined the potential role of the estrous cycle on sucrose self-administration or stereotyped behaviors. We address these gaps in knowledge by including males and females and by tracking the estrous cycle throughout in our assessments of intake, craving, and other addiction-related behaviors.

The overarching goal of this work was to delineate and fine-tune the more subtle changes in behavior and affective state that accompany increased drug seeking in response to cue re-exposure. We aimed to identify behaviors that accompany craving generally. Another objective of this study was to identify drug-specific behavioral correlates of craving that do not generalize to natural rewards. This method provides important insight into characteristics of "normal" (non-pathological) craving behaviors versus those that specifically accompany heroin craving. Furthermore, sucrose comparisons can aid in differentiating the mechanisms of cuereward associations and memory consolidation broadly from those that govern opioid-paired cue-induced craving. These findings can 1) improve the preclinical model of opioid craving/relapse-like like behavior and 2) lay the groundwork for identification of circuits governing opioid-cue reactivity. Males and females were included to address potential sex differences. All measurements were tracked across the estrous cycle with the goal of identifying whether fluctuations in gonadal hormones affect self-administration, craving, and/or any of the 
more nuanced behaviors. These findings contribute to a better behavioral definition of craving, and aid in identifying opioid-specific neural circuits involved in craving.

\section{Methods}

$\underline{\text { Animals }}$

For all experiments, male $(n=51)$ and female $(n=66)$ Long-Evans rats were bred in house. All animals used in the studies were 60-180 days old and were pair-housed. Rats were housed in a reverse light-dark cycle colony room with lights off at 9:00AM and were handled daily for 2-5 minutes each for at least five days prior to the start of any behavioral procedure or test. Experiments were conducted in the dark phase. Animals had ad libitum access to water and standard laboratory chow throughout the experiment. All animal procedures were performed in accordance with the [Author University] animal care committee's regulations. Heroin self-administration, forced abstinence, and relapse tests

Rats used for intravenous heroin self-administration were anesthetized using an intraperitoneal injection of $80 \mathrm{mg} / \mathrm{kg}$ ketamine and $12 \mathrm{mg} / \mathrm{kg}$ xylazine prior to surgery. An indwelling silastic catheter was threaded subcutaneously over the shoulder blade, inserted in the jugular vein, and sutured in place. The catheter routed to a mesh back mount platform (Strategic Applications Inc., Lake Villa, IL), which was sutured below the skin between the shoulder blades. Catheters were flushed daily with $0.2 \mathrm{~mL}$ of timentin $(0.93 \mathrm{mg} / \mathrm{mL})$ dissolved in heparinized saline to prevent clogging. Following the 7-day recovery period, rats were placed in operant chambers and allowed to lever press on a fixed ratio 1 (FR1) schedule for heroin infusions $(0.10 \mathrm{mg} / \mathrm{kg} /$ infusion over 5 seconds). Responses on the "active lever" resulted in one infusion, accompanied by a 5 -second light cue and a subsequent 20 -second timeout period during which the house light was off and lever presses were recorded but had no contingent drug infusion. Sessions began at the start of the animals' dark cycle (9:00 AM). Heroin self-administration sessions followed an intermittent access paradigm, which included three hours of "drug-available" time, followed by a 15-minute window of "no-drug 
available". This procedure repeated three times per session, resulting in a total of nine hours of drug availability, and was performed daily on 10 consecutive days. Heroin self-administering rats were tested behaviorally for signs of craving during early (Day 1) and late (Day 30) abstinence using a within-subjects design. Within-subjects relapse tests ("cue tests") began at the start of the animals' dark cycle and were 30 minutes long under extinction conditions, meaning that active lever presses resulted in cue light presentation, but no contingent reward. Throughout abstinence (one or 30 days), rats were housed in their home cage, were not reexposed to the self-administration chambers, and were handled regularly. $\underline{\text { Sucrose self-administration, forced abstinence, and relapse tests }}$

Separate cohorts of male and female rats self-administered sucrose. This natural, nondrug reward acts as an important control group in establishing baseline behaviors that change over 30 days of forced abstinence. Unsurgerized drug-naive rats responded on the "active lever" for one sucrose pellet (FR1 schedule) that was accompanied by a 5 -second light cue, and a subsequent 20 -second timeout period as previously described. Sucrose self-administration began at the start of the animals' dark cycle and occurred for two hours daily on 10 consecutive days. At the end of one or 30 days of forced abstinence (as described above), male and female rats were tested for sucrose seeking for one hour.

\section{$\underline{\text { Recording relapse tests and scoring for stereotyped behaviors }}$}

Each cue test was video recorded for off-line scoring by investigators blind to condition. Videos were recorded in 5-minute increments via SD card dashcams (Pruveeo, Blaine, WA) attached to the top of each sound-attenuating chamber to provide an aerial view of the operant box floor. Raw footage was then edited together using Adobe Premiere Pro (Adobe Inc., San Jose, CA) to create one continuous video.

Scored behaviors included horizontal locomotion and stereotypies. These were tracked using an online counter and timer. To measure horizontal locomotion, a two by two grid was superimposed over the floor of the operant chamber displayed on the computer screen in the 
221

222

223

224

225

226

227

228

229

230

231

232

233

234

235

236

237

238

239

240

241

242

243

244

245

recording. The grid was measured to ensure that it was approximately $15 \mathrm{~cm}$ by $15 \mathrm{~cm}$ and that each quadrant was of equal size. Scorers then counted each "beam break", or instance in which the rat moved from one quadrant of the chamber to another by crossing one of the grid lines with its head and shoulders. Time spent grooming was quantified by the amount of time an animal spent licking, pawing, or scratching itself. Time spent sniffing was quantified by the amount of time that an animal's nose was in contact with the floor or walls of the operant box, either while stationary, or while mobile. The number of hops or darts across the operant chamber quantified instances of hyperactivity.

Audio recording and scoring ultrasonic vocalizations (USVs)

Ultrasonic vocalizations (USVs) were collected from a subset of rats during cue tests.

Ultrasonic microphones from Dodotronic (Ultramic 200K) were placed atop the selfadministration chamber and positioned inside a foam noodle to secure them in an upright position. Microphones were plugged into HP computers and USV recordings were obtained using Raven Pro 1.5 beta (Ithaca, NY) at a 192,000-Hz sampling rate in 10-minute segments. WAVE files were run through DeepSqueak, a Matlab-based USV detection program.

The Short Rat Call neural network was used to detect $50-\mathrm{kHz}$ USVs. Calls were accepted or rejected based on if they met frequency criteria $(38-90 \mathrm{kHz})$ and duration criteria (5 ms or longer). Calls were differentiated from background noise by visual confirmation of a clear shape on the spectrogram. The Long Rat Call Network was used to detect 22-kHz USVs within the range of $18-33-\mathrm{kHz}$. Trained scorers counted the number of USVs during the duration of the cue test. If needed, calls were verified via playback in DeepSqueak. Calls in the 18-33-kHz range were termed "22-kHz USVs" and were interpreted as a negative-valence affective state. Calls in the 38-90-kHz range were termed "50-kHz USVs" and were interpreted as a positivevalence affective state (Barker et al., 2015).

Gonadal hormone measures 

administration, vaginal lavage was conducted daily to collect loose vaginal cells. Lavage data was collected for a minimum of ten consecutive days prior to, as well as the day of each cue test. Vaginal cytology was conducted in order to determine the concentration and volume of three main cell types associated with distinct phases of the female estrous cycle: diestrus, as indicated by an abundance of sparse leukocytes, proestrus, as indicated by nucleated epithelial nuclei, and estrus, as indicated by dense, overlapping cornified cells. For the purposes of these experiments, the cycle was split into estrus (proestrus and estrus) and non-estrus (metestrus and diestrus) phases.

To determine the effect of the female estrous cycle on sucrose and/or heroin selfadministration, the average number of pellets/infusions earned was calculated and compared across all female rats in estrus versus non-estrus during each day of self-administration. During the early and late abstinence cue tests, the number of active lever presses was averaged and compared for female rats in estrus versus those in non-estrus. Overall locomotion, sniffing, grooming, and hyperactivity were compared for each rat individually and grouped according to whether a given rat was in estrus or non-estrus on the day of the seeking test. Infusions/pellets earned were used as a proxy for opioid/sucrose consumption, whereas active lever presses during the cue test were used to interpret craving.

\section{Statistical Analysis}

Three-way ANOVAs were used to compare active lever responses during the Day 1 or Day 30 cue test with abstinence condition (Day 1 vs Day 30 ), sex, and reinforcer (sucrose vs heroin) as between-subjects factors. Three-way ANOVAs were used for overall locomotion, sniffing, grooming, hyperactivity, and $50-\mathrm{kHz}$ ultrasonic vocalization analyses with sex, reinforcer, and abstinence condition as between-subjects factors. In all cases, if a significant interaction was found after performing ANOVAs, post-hoc comparisons were made using

271 Bonferroni's multiple comparisons test. 
For comparisons of heroin and sucrose taking across phases of the estrous cycle, mixed

273

274

275 model ANOVAs were used to compare infusions/pellets earned during self-administration. Session (self-administration day 1 vs day 2 vs day 3 etc.) was used as the within-subjects factor, and condition (estrus vs non-estrus) was used as the between-subjects factor. Two-way repeated-measures ANOVAs were used to compare active lever presses during the early (Day 1) and late (Day 30) abstinence cue tests. Abstinence condition (abstinence Day 1 vs abstinence Day 30) and estrous cycle phase (estrus vs non-estrus) were used as betweensubjects factors. Again, if a significant interaction was found after performing ANOVAs, post-hoc comparisons were made using Sidak's or Bonferroni's multiple comparisons test. For comparisons of locomotion, sniffing, grooming, and hyperactivity in estrus versus non-estrus females in early and late abstinence, a two-way ANOVA was used. Abstinence day and estrous cycle phase were both between-subjects factors. Sidak's and Bonferroni's multiple comparisons tests were used if an interaction was detected.

\section{Results}

Incubation of sucrose and heroin craving are associated with reinforcer-specific behavioral profiles

Male and female rats were allowed to intravenously self-administer (IVSA) heroin $(0.10 \mathrm{mg} / \mathrm{kg} / \mathrm{infusion})$ or self-administer sucrose pellets for 10 days, and were then assigned to either one (Day 1) or 30 days (Day 30) of forced abstinence. At the end of each respective abstinence time point, rats were tested for drug- or sucrose-seeking behavior. (Figure 1A). A camera was mounted to the ceiling of the sound-attenuating chamber and an ultrasonic microphone was positioned in the front right corner atop the Plexiglass ceiling of the operant chamber to score behaviors and record ultrasonic vocalizations (USVs), respectively (Figure 1B). Active lever responses increased from abstinence Day 1 to abstinence Day 30 in heroinand sucrose-treated males and females, indicating incubation of craving (Figure 1C; additional statistics in Table 1; day $\left.\left[F_{1,232}=52.74, p<.0001\right]\right)$. Overall locomotion, as measured by the total 
number of times a rat crossed one of the superimposed beams bisecting the operant chamber in the video recording, increased over 30 days of abstinence from both heroin and sucrose in both sexes (Figure 1D; additional statistics in Table 1; day [ $\left.\left.F_{1,232}=100.6, p<.0001\right]\right)$. Grooming decreased in heroin males and females, and was unchanged in sucrose males and females after 30 days of abstinence (Figure 1E; additional statistics in Table 1; interaction $\left[F_{1,232}=4.318, p=.0388\right]$; Day 1 male heroin vs Day 30 male heroin, $p=.0138$; Day 1 male sucrose vs Day 30 male sucrose, $p>$.9999; Day 1 female heroin vs Day 30 female heroin, $p<.0001$; Day 1 female sucrose vs Day 30 female sucrose, $p=.1848$, Bonferroni's multiple comparisons test). Sniffing was unchanged in all conditions, with the exception of an increase in sucrose-exposed females (Figure 1F; additional statistics in Table 1; interaction $\left[F_{1,232}=4.304, p=.0391\right]$; Day 1 male heroin vs Day 30 male heroin, $p>.9999$; Day 1 male sucrose vs Day 30 male sucrose, $p=.1316$; Day 1 female heroin vs Day 30 female heroin, $p=.1848$; Day 1 female sucrose vs Day 30 female sucrose, $p<.0001$, Bonferroni's multiple comparisons test). Hyperactivity, as measured by hops and darts across the cage, increased over 30 days of abstinence from heroin, but not sucrose in males and females (Figure 1G; additional statistics in Table 1; interaction $\left[F_{1,232}=12.11, p=.0006\right]$; Day 1 male heroin vs Day 30 male heroin, $p=.0050$; Day 1 male sucrose vs Day 30 male sucrose, $p>.9999$; Day 1 female heroin vs Day 30 female heroin, $p<.0001$; Day 1 female sucrose vs Day 30 female sucrose, $p=.7376$, Bonferroni's multiple comparisons test). 
A

Sucrose

or Heroin

Self-Administration Abstinence

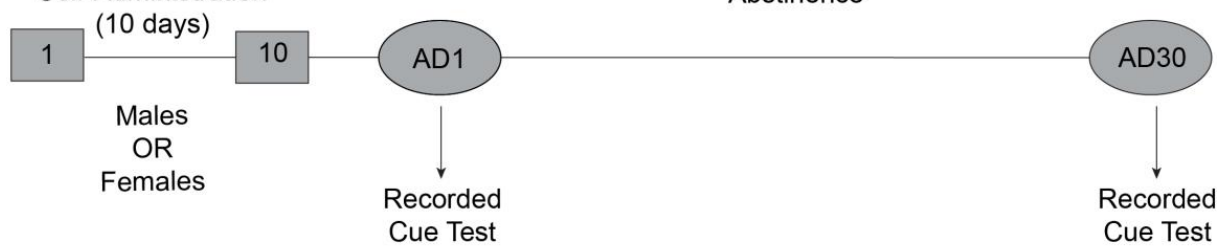

B

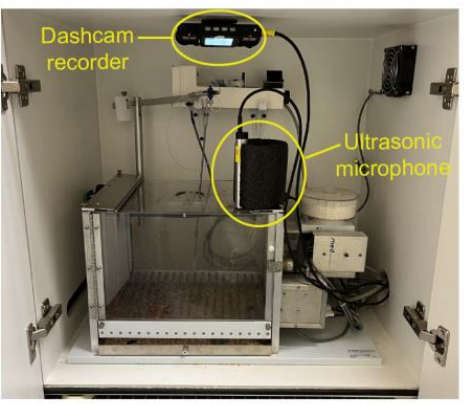

C $\bullet \bullet \bullet$ Day 30

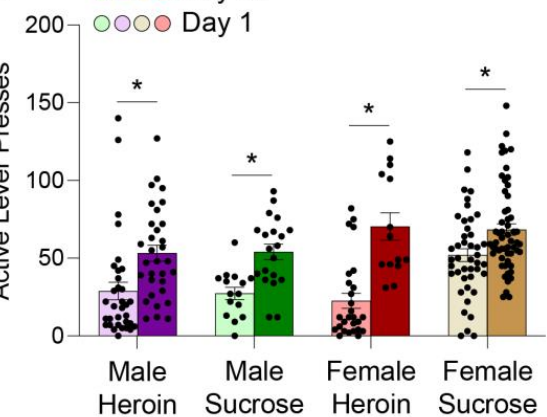

D

E
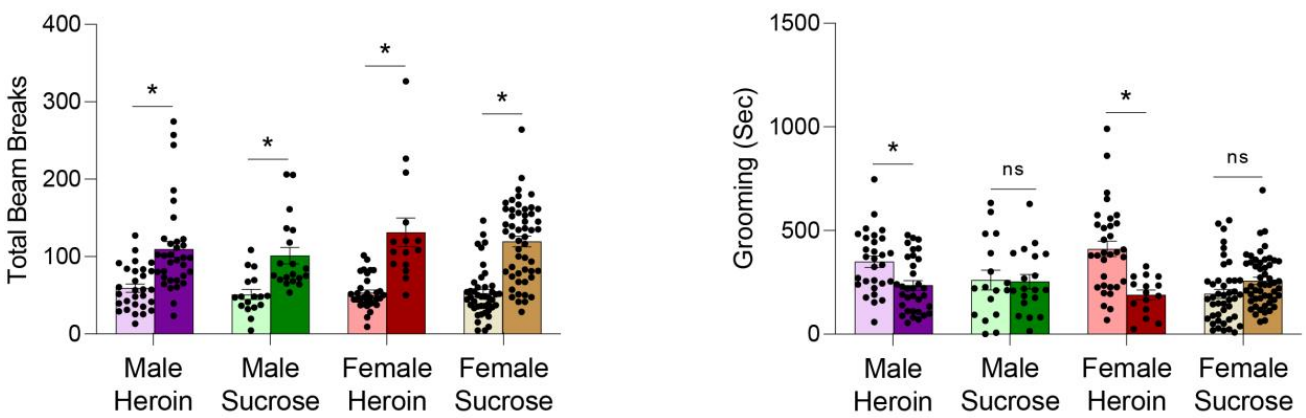

$\mathbf{F}$

G
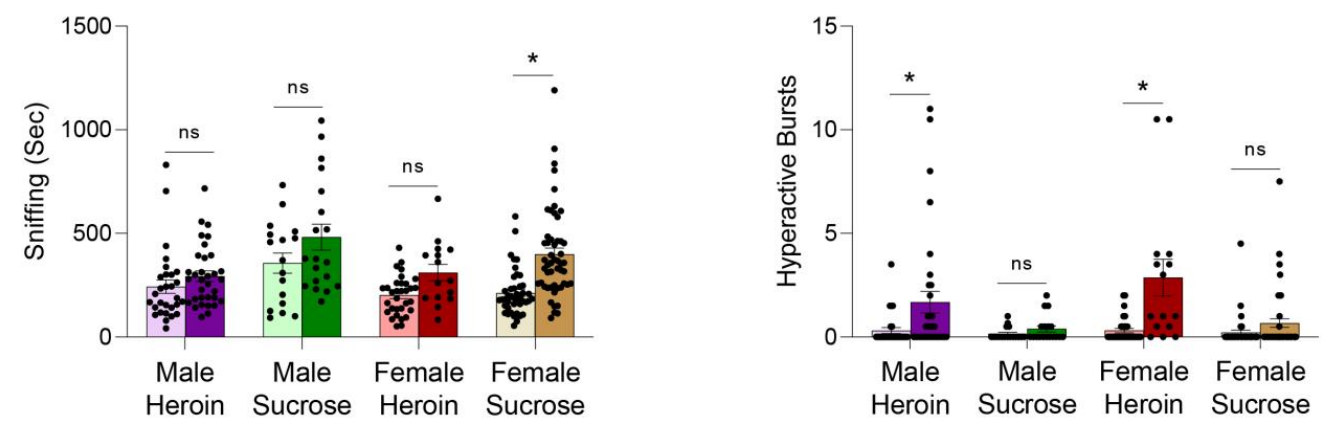
Figure 1. Incubation of craving is associated with sex- and reinforcer-specific behavioral signatures. A. Experimental timeline including 10 days of intravenous heroin or oral sucrose self-administration, followed by a recorded cue test after one or 30 days of forced abstinence. B. Cameras were mounted on the ceiling of the sound-attenuating chamber to record movement and behaviors during the cue test. An ultrasonic microphone was positioned flush against the Plexiglas top of the operant box to record ultrasonic vocalizations. C. Sucrose- and heroin-exposed males and females showed incubation of craving, as indicated by an increase in active levers after 30 days of abstinence compared to one day of abstinence. Post-hoc comparisons revealed that sucrose females responded significantly more than heroin females and sucrose males in early abstinence (day $\left[F_{1,232}=52.74, p<.0001\right]$; Day 1 female sucrose vs Day 1 female heroin, $p=.0003$; Day 1 female sucrose vs Day 1 male sucrose, $p=.0402$, Bonferroni's multiple comparisons test). D. Overall locomotion, as measured by total beam breaks increased in heroin- and sucrose-exposed males and females over 30 days of abstinence (day $\left.\left[F_{1,232}=100.6, p<.0001\right]\right)$. E. Grooming decreased in heroin-exposed rats and was unchanged in sucrose-exposed rats of both sexes after 30 days of abstinence (interaction $\left[F_{1,232}=4.318, p=.0388\right]$; Day 1 male heroin vs Day 30 male heroin, $p=.0138$; Day 1 male sucrose vs Day 30 male sucrose, $p>$.9999; Day 1 female heroin vs Day 30 female heroin, $p<.0001$; Day 1 female sucrose vs Day 30 female sucrose, $p=.1848$, Bonferroni's multiple comparisons test). F. Sniffing was unchanged in all conditions, with the exception of an increase in sucrose-exposed females. Post-hoc comparisons revealed that sucrose males engaged in more sniffing than heroin males in late abstinence, and more sniffing than sucrose females in early abstinence (interaction $\left[F_{1,232}=4.304, p=.0391\right]$; Day 1 male heroin vs Day 30 male heroin, $p>.9999$; Day 1 male sucrose vs Day 30 male sucrose, $p=.1316$; Day 1 female heroin vs Day 30 female heroin; Day 1 female sucrose vs Day 30 female sucrose, $p<.0001$, $p=.1848$, Bonferroni's multiple comparisons test). G. Hyperactivity, as measured by hops and darts across the cage, increased over 30 days of abstinence from heroin, but not sucrose in males and females (interaction $\left[F_{1,232}=12.11, p=.0006\right]$; Day 1 male heroin vs Day 30 male heroin, $p=.0050$; Day 1 male sucrose vs Day 30 male sucrose, $p>$.9999; Day 1 female heroin vs Day 30 female heroin, $p<.0001$; Day 1 female sucrose vs Day 30 female sucrose, $p=.7376$, Bonferroni's multiple comparisons test). Additional statistics are listed in Table 1. Data show mean \pm SEM; ${ }^{*} p<.05$.

\section{Heroin-exposed male rats show an increase in $50-\mathrm{kHz}$ USVs after 30 days of forced abstinence}

22-kHz USVs are unchanged in heroin-exposed males and females (data not shown).

$32550-k H z$ USVs increased only in heroin-exposed male rats and were unchanged in all other

326 groups after 30 days of abstinence (Figure 2; additional statistics in Table 1; interaction

$327\left[F_{1,51}=60.12, p<.0001\right]$; Day 1 heroin male $50-\mathrm{kHz}$ calls vs Day 30 heroin male $50-\mathrm{kHz}$ calls,

$328 p<.0001$; Day 1 sucrose male $50-\mathrm{kHz}$ calls vs Day 30 sucrose male $50-\mathrm{kHz}$ calls, $p>.9999$; Day

3291 heroin female $50-\mathrm{kHz}$ calls vs Day 30 heroin female $50-\mathrm{kHz}$ calls, $p>.9999$; Day 1 sucrose

330 female $50-\mathrm{kHz}$ calls vs Day 30 sucrose female $50-\mathrm{kHz}$ calls, $p>.9999$, Bonferroni's multiple

331 comparisons test). Very few $22-\mathrm{kHz}$ calls were observed during any of the cue tests. Many were

332 short in length and captured in conjunction with a series of $50-\mathrm{kHz}$ calls. Compared to $50-\mathrm{kHz}$ 


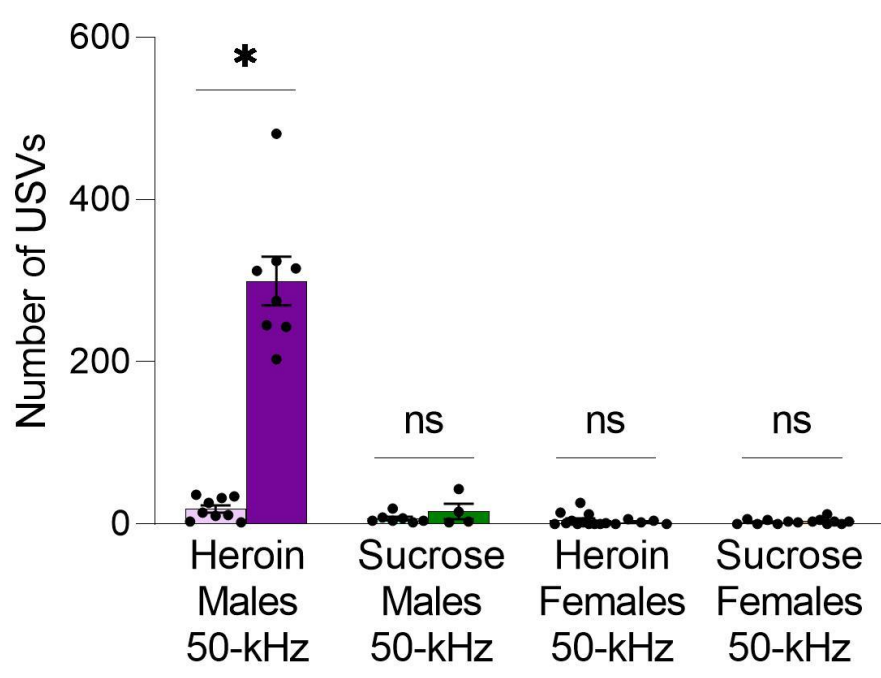

Figure 2. Incubation of heroin craving is associated with an increase in $50-\mathrm{kHz}$ ultrasonic vocalizations (USVs) in males only (interaction $\left[F_{1,51}=60.12, p<.0001\right]$; Day 1 heroin male $50-\mathrm{kHz}$ calls vs Day 30 heroin male $50-\mathrm{kHz}$ calls, $p<.0001$; Day 1 sucrose male $50-\mathrm{kHz}$ calls vs Day 30 sucrose male $50-\mathrm{kHz}$ calls, $p>$.9999; Day 1 heroin female 50-kHz calls vs Day 30 heroin female $50-\mathrm{kHz}$ calls, $p>.9999$; Day 1 sucrose female $50-\mathrm{kHz}$ calls vs Day 30 sucrose female $50-\mathrm{kHz}$ calls, $p>.9999$, Bonferroni's multiple comparisons test). Additional statistics are listed in Table 1. Data show mean \pm SEM; ${ }^{*} p<.05$.

339

340

341

342

343

344

345

346

347

348

349

\section{Estrous cycle does not impact heroin or sucrose self-administration nor incubation of craving}

Over ten days of self-administration females took increasing amounts of heroin (Figure 3A; time, $\left[F_{9,541}=3.821, p=.0001\right]$, interaction $\left.\left[F_{27,541}=4.405, p<.0001\right]\right)$. There was no difference in heroin taking between females in estrus versus those in non-estrus (Figure 3A; estrus, $\left.\left[F_{3,148}=.5299, p=.6624\right]\right)$. Female rats experienced incubation of heroin craving following 30 days of abstinence (Figure 3B; time, $\left[F_{1,51}=18.02, p<.0001\right]$ ); however, craving was not affected by phase of the estrous cycle (Figure 3B; estrus, [ $\left.F_{1,51}=.0881, p=.7677\right]$; interaction, 
$\left.350\left[F_{1,51}=1.989, p=.1645\right]\right)$. Similarly, increasing amounts of sucrose were taken over 10 days of

351 self-administration (Figure $3 \mathbf{C}$; time, $\left.\left[F_{9,760}=66.68, p<.0001\right]\right)$, and there were no differences

352 between females in estrus versus those in non-estrus (Figure 3C; estrus, [ $F_{1,201}=.2342$,

$353 p=.6290]$; interaction, $\left.\left[F_{9,760}=.8406, p=.5787\right]\right)$. Additionally, females experienced incubation of

354 craving for the natural reward sucrose (Figure 3D; time [ $\left.F_{1,63}=12.57, p=.0007\right]$ ), and there was

355 no effect of estrous cycle phase on sucrose craving (Figure 3D; estrus, [ $\left.F_{1,63}=.2057, p=0.6517\right]$;

356 interaction, $\left.\left[F_{1,63}=.7248, p=.6517\right]\right)$.

357

A
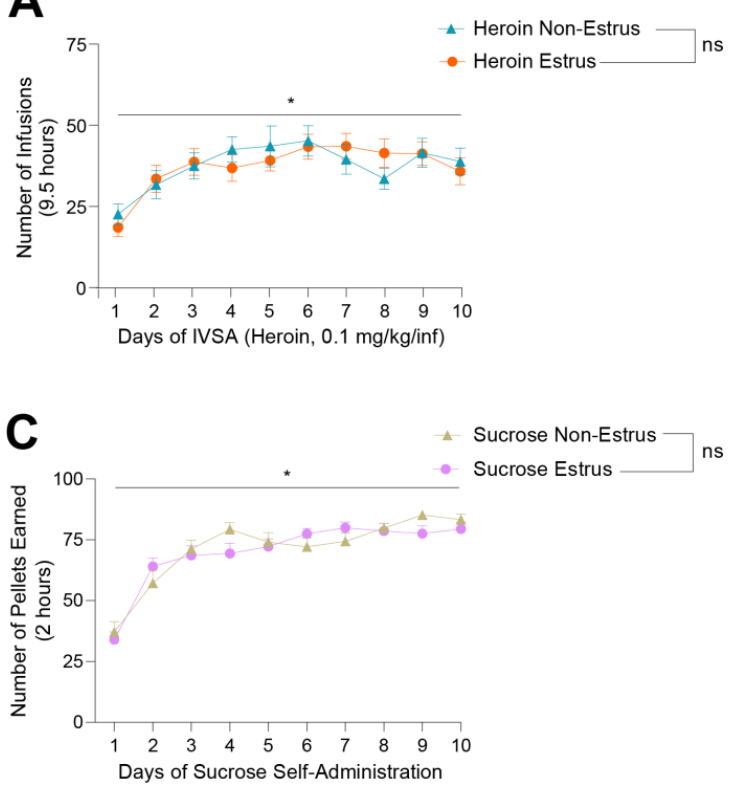

B

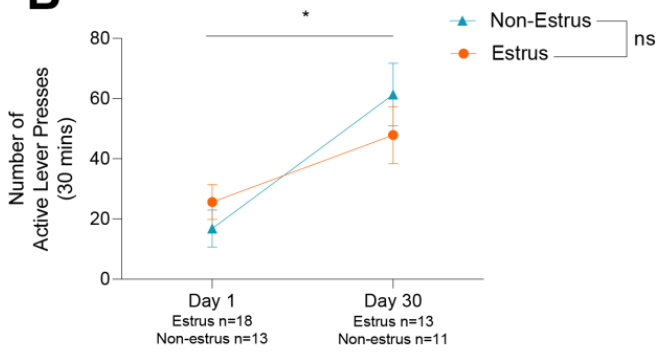

D

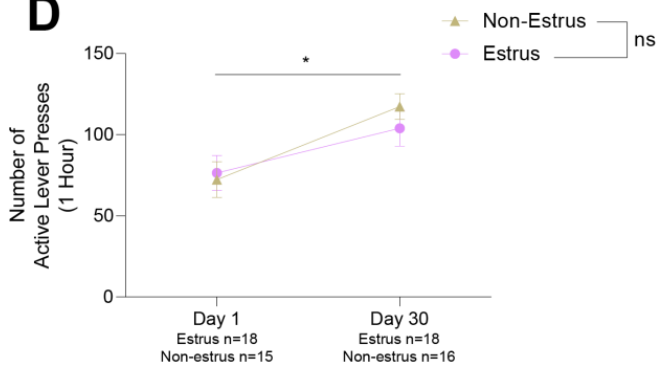


Figure 3. The effect of the female estrous cycle phase (estrus versus non-estrus) on heroin and sucrose self-administration and craving. A. Female heroin-treated rats earned more infusions over 10 days of intravenous self-administration regardless of estrous cycle phase (time, $\left[F_{9,541}=3.821, p=.0001\right]$, estrus, $\left[F_{3,148}=.5299, p=.6624\right]$; interaction, $\left.\left[F_{27,541}=4.405, p<.0001\right]\right)$. B. Females demonstrated incubation of heroin craving, as indicated by an increase in active lever responses during a 30-minute cue test following 30 days of abstinence, and there were no differences in active lever responses between estrus and non-estrus females at either abstinence time point (time, $\left[F_{1,51}=18.02, p<.0001\right]$, estrus, $\left[F_{1,51}=.0881, p=.7677\right]$; interaction, $\left.\left[F_{1,51}=1.989, p=.1645\right]\right)$. C. Female sucrose-treated rats earned more sucrose pellets over 10 days of oral self-administration regardless of estrous cycle phase (time, $\left[F_{9,760}=66.68, p<.0001\right]$, estrus, $\left[F_{1,201}=.2342, p=.6290\right]$; interaction, $\left.\left[F_{9,760}=.8406, p=.5787\right]\right)$. D. Females demonstrated incubation of sucrose craving, as indicated by an increase in active lever responses during a 1-hour cue test following 30 days of abstinence, and there were no differences in active lever responses between estrus and non-estrus females at either abstinence time point (time, $\left[F_{1,63}=12.75, p=.0007\right]$, estrus, [ $\left.F_{1,63}=.2057, p=.6517\right]$; interaction, $\left.\left[F_{1,63}=.7248, p=.6517\right]\right)$. Data show mean \pm SEM; * $p<.05$.

360

361

362

363

364

365

366

367

368

369

370

371

372

373

374

375

376

Locomotion is the only behavior enhanced during estrus females after 30 days of abstinence from heroin compared to non-estrus

Total beams breaks increased from Day 1 to Day 30 of abstinence in heroin-treated

females (Figure 4A; time $\left.\left[F_{1,45}=33.58, p<.0001\right]\right)$. Estrus females showed higher locomotion than

non-estrus females (Figure 4A; estrus, $\left[F_{1,45}=6.151, p=.0169\right]$; interaction,

$\left.\left[F_{1,45}=.5535, p=.4608\right]\right)$. Heroin females experienced a decrease in grooming over 30 days of

abstinence that was not affected by phase of the estrous cycle (Figure 4B; time,

$\left[F_{1,45}=18.30, p=.0001\right]$; estrus, $\left[F_{1,45}=.0093, p=.9235\right]$; interaction, $\left.\left[F_{1,45}=1.762, p=.1911\right]\right)$. Sniffing

increased over 30 days of abstinence from heroin regardless of phase of the estrous cycle

(Figure 4C; time, $\left[F_{1,45}=9.245, p=.0039\right]$; estrus, $\left[F_{1,45}=1.018, p=.3183\right]$; interaction,

$\left.\left[F_{1,45}=.0221, p=.8826\right]\right)$. Interestingly, the increase in hyperactivity over 30 days of abstinence

from heroin was not affected by the phase of the estrous cycle (Figure 4D; time,

$\left[F_{1,45}=15.36, p=.0003\right]$; estrus, $\left[F_{1,45}=.0558, p=.8144\right]$; interaction, $\left.\left[F_{1,45}=.2112, p=.6481\right]\right)$. 
A

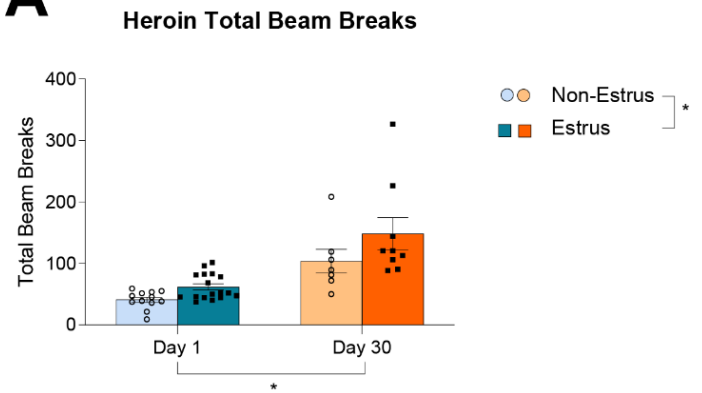

C
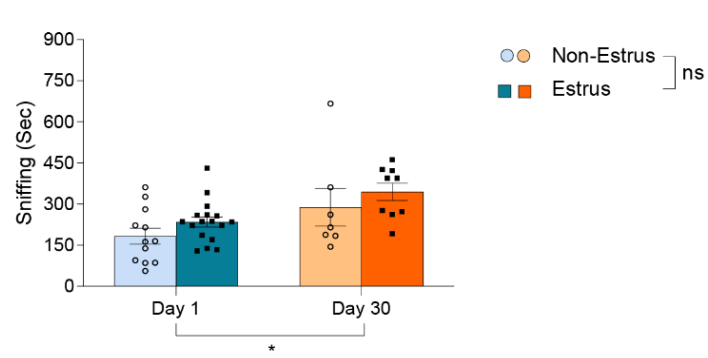

घ- Estrus
B

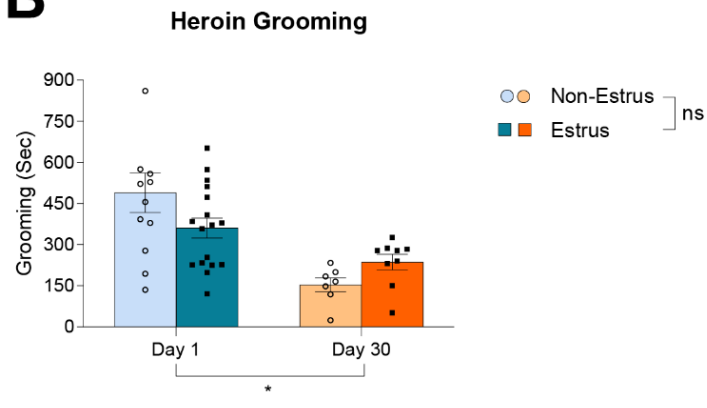

D

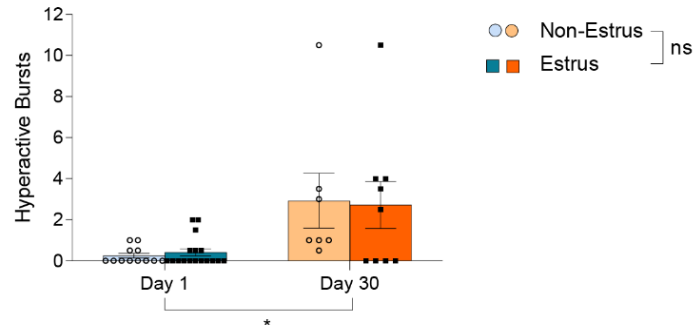

377

Figure 4. The effect of the female estrous cycle phase (estrus versus non-estrus) on addiction-related behaviors following one or 30 days of abstinence from heroin. A. Total beam breaks increased over 30 days of abstinence, and were enhanced in estrus females compared to non-estrus females (time

$\left[F_{1,45}=33.58, p<.0001\right]$; estrus, $\left[F_{1,45}=6.151, p=.0169\right]$; interaction, $\left.\left[F_{1,45}=0.5535, p=.4608\right]\right)$. B. Grooming decreased over 30 days of abstinence regardless of estrous cycle phase (time, [ $\left.F_{1,45}=18.30, p=.0001\right]$; estrus, $\left[F_{1,45}=0.0093, p=.9235\right]$; interaction, $\left.\left[F_{1,45}=1.762, p=.1911\right]\right)$. C. Sniffing increased over 30 days of abstinence regardless of estrous cycle phase (time, $\left[F_{1,45}=9.245, p=.0039\right]$; estrus, $\left[F_{1,45}=1.018, p=.3183\right]$; interaction, $\left.\left[F_{1,45}=0.0221, p=.8826\right]\right)$. D. Hyperactivity increased over 30 days of abstinence and there was no effect of estrous cycle phase (time, $\left[F_{1,45}=15.36, p=.0003\right]$; estrus, $\left[F_{1,45}=0.0558, p=.8144\right]$; interaction, $\left.\left[F_{1,45}=0.2112, p=.6481\right]\right)$. Data show mean \pm SEM; ${ }^{*} p<.05$.

Locomotion and hyperactivity are enhanced in estrus females after 30 days of abstinence from sucrose

As with heroin-treated females, overall locomotion, as measured by total beam breaks increased over 30 days of abstinence from sucrose (Figure 5A; time, $\left[F_{1,86}=54.91, p<.0001\right]$ ).

Again, locomotion was enhanced in estrus females compared to non-estrus females (Figure

5A; estrus, $\left[F_{1,86}=6.386, p=.0133\right]$; interaction, $\left.\left[F_{1,86}=.0115, p=.9150\right]\right)$. Grooming increased over 
38730 days of abstinence from sucrose (Figure $5 \mathbf{B}$; time $\left[F_{1,86}=4.194, p=.0436\right]$ ), but was not

388 affected by phase of the estrous cycle (Figure 5B; estrus, [ $\left.F_{1,86}=1.091, p=.2993\right]$; interaction,

$\left.389\left[F_{1,86}=1.974, p=.11636\right]\right)$. Similarly, sniffing increased over 30 days of abstinence from sucrose

390 (Figure 5C; time $\left.\left[F_{1,86}=23.98, p<.0001\right]\right)$, but was not affected by phase of the estrous cycle

391 (Figure 5C; estrus, $\left[F_{1,86}=0.003, p=.9568\right]$; interaction, $\left.\left[F_{1,86}=.064, p=.8006\right]\right)$. Hyperactivity was

392 enhanced in estrus females at Day 30 of abstinence, but was not affected by estrous cycle

393 phase in early abstinence (Figure 5D; time, $\left[F_{1,86}=3.923, p=.0508\right]$; estrus, $\left[F_{1,86}=5.459, p=.0218\right]$;

394 interaction, [ $\left.F_{1,86}=1.767, p=.1872\right]$; Day 1 estrus vs non-estrus, $p>.9999$; Day 30 estrus vs non-

395 estrus, $p=.0479$, Bonferroni's multiple comparisons test).

396

A sucrose Total Beam Breaks

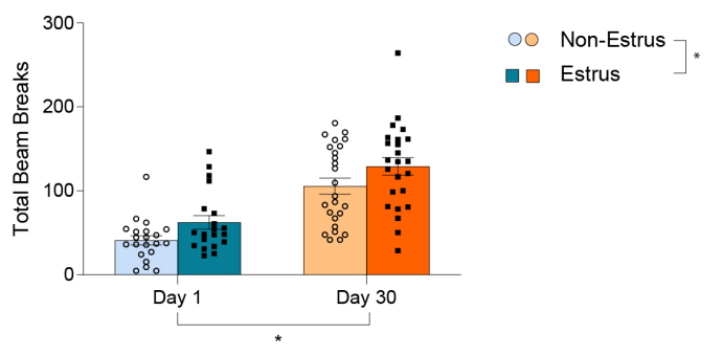

C

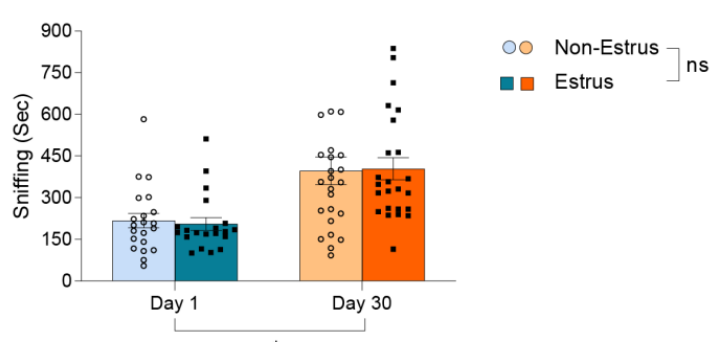

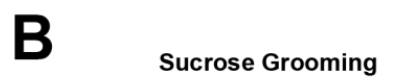

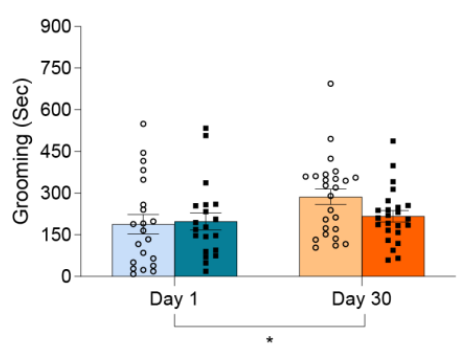

○० Non-Estrus 7 ns घ- Estrus
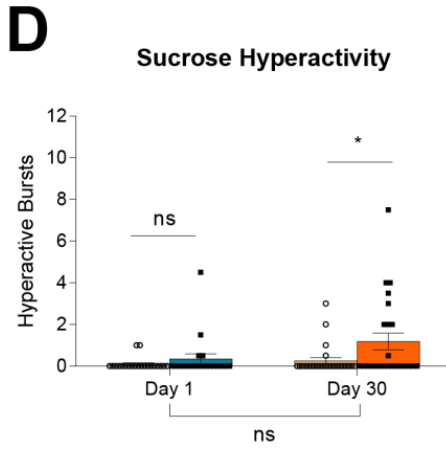

○० Non-Estrus 7 .

a Estrus 
Figure 5. The effect of the female estrous cycle phase (estrus versus non-estrus) on addiction-related behaviors following one or 30 days of abstinence from sucrose. A. Total beam breaks increased over 30 days of abstinence, and were enhanced in estrus females compared to non-estrus females (time, $\left[F_{1,86}=54.91, p<.0001\right]$; estrus, $\left[F_{1,86}=6.386, p=.0133\right]$; interaction, $\left.\left[F_{1,86}=0.0115, p=.9150\right]\right)$. B. Grooming increased over 30 days of abstinence regardless of estrous cycle phase (time $\left[F_{1,86}=4.194, p=.0436\right]$ ); estrus, $\left[F_{1,86}=1.091, p=.2993\right]$; interaction, $\left.\left[F_{1,86}=1.974, p=.11636\right]\right)$. C. Sniffing increased over 30 days of abstinence regardless of estrous cycle phase (time $\left[F_{1,86}=23.98, p<0.0001\right.$; estrus, $\left[F_{1,86}=0.003, p=.9568\right]$; interaction, $\left.\left[F_{1,86}=0.064, p=.8006\right]\right)$. D. Hyperactivity was enhanced at Day 30 in estrus females, and there was no effect of estrous cycle on hyperactivity at Day 1 (time, [ $\left.F_{1,86}=3.923, p=.0508\right]$; estrus, $\left[F_{1,86}=5.459, p=.0218\right]$; interaction, $\left[F_{1,86}=1.767, p=.1872\right]$; Day 1 estrus vs non-estrus, $p>0.9999$; Day 30 estrus vs non-estrus, $p=0.0479$, Bonferroni's multiple comparisons test). Data show mean $\pm \mathrm{SEM} ;{ }^{*} p<.05$.

402

403

404

405

406

407

408

409

410

411

412

413

414

415

416

417

418

419

420

421

422

423

\section{Discussion}

This study aimed to refine a more robust picture of behavioral and affective states that accompany opioid seeking after extended abstinence, when craving is high. After chronic selfadministration of either intravenous heroin or the natural reward sucrose, rats underwent an abstinence paradigm that is known to induce craving after 30 days (Lu et al., 2004). Sucrose is a natural reward that acts on many of the same brain regions as drugs of abuse and leads to enhanced craving. Thus, it was important to establish behaviors that change in response to extended abstinence and cue re-exposure generally, and subsequently to differentiate opioidspecific behavioral changes. To that end, we explored overall locomotion, sniffing, grooming, hyperactivity, ultrasonic vocalizations (USVs), and estrous cycle phase. Results indicate a reinforcer-specific behavioral profile that emerges after 30 days of forced abstinence in heroinexposed rats. Interestingly, total beam breaks increased over 30 days of abstinence regardless of sex and reinforcer, indicating that increased craving is associated with a general increase in locomotion. Heroin exposure resulted in a unique behavioral signature, including a decrease in grooming and an increase in hyperactivity that was seen in both sexes but did not generalize to the natural reward sucrose. Sniffing showed a sex- and reinforcer-specific effect, such that it increased in sucrose-treated females over 30 days of abstinence, and was unchanged in all other groups. Heroin-exposed males made significantly more $50-\mathrm{kHz}$ USVs during the late 
424 abstinence seeking test when craving was high, which was not seen in females, nor sucroseexposed rats of either sex. Estrous cycle phase did not influence self-administration nor craving

426 for either reinforcer. However, locomotion and/or hyperactivity were enhanced in estrous females in a reinforcer-specific manner. These results underscore the notion that opioids cause long-lasting neural adaptations, which take effect and persist throughout abstinence in order to

429 prime the system for increased craving and compulsive drug seeking when re-exposed to drugpaired cues. These changes are characterized by decreased grooming and increased hyperactivity, which represent an opioid-specific behavioral profile of craving in response to cue re-exposure. An increase in positive-valence affective state is thought to drive this drug-seeking behavioral signature, though this may be sex-specific. The mechanism for explaining the different behavioral profiles in heroin- versus sucrose-exposed rats is undetermined and outside the scope of this report. Ultimately, the goal is that these descriptive data lay the foundation for further exploration of such a mechanism. Taken together, these results are promising in terms of developing treatments tailored to opioid craving and associated behaviors without interrupting the natural reward system. but also a general increase in overall locomotion, as measured by beam breaks. There are similarities between changes in locomotion/stereotypies and incubation of craving, such that both persist after long periods of abstinence (Paulson et al., 1991; Pickens et al., 2011; Madangopal et al., 2019). For example, locomotion increases during a cue-induced reinstatement test following chronic sucrose self-administration and 30 days of abstinence (Grimm et al., 2007; Aoyama et al., 2014; Harkness et al., 2016), though in some of these studies, rats had undergone extinction training prior to reinstatement. Following exposure to experimenter-delivered morphine, a priming injection stimulated increased locomotor 
451 sensitization after three and nine weeks of abstinence (Vanderschuren et al., 1997). Others

452 have shown that this persists for up to three months of abstinence (Powell and Holtzman, 2001).

453 Though these studies (and the bulk of studies examining sensitization) focus on changes with

454 opioids on board, one report found that overall locomotion is depressed 24 hours into

455 abstinence without re-exposure to heroin. Although it is unclear whether this change reached

456 significance, locomotion appears to increase following 14 days of abstinence, again without a

457 priming injection (Leri et al., 2003). All of these aforementioned conclusions were based on

458 male subjects only. This study corroborates these findings and extends them to females.

$459 \quad$ Locomotion and reinstatement are mediated by overlapping neurotransmitter

460 mechanisms in the ventral tegmental area and accumbens, whereby activation of glutamate

461 receptors on accumbal dopamine terminals leads to the increase in dopamine that drives

462 increased locomotion and motivation (Wu et al., 1993; Wise, 2004). In fact, reducing locomotion

463 and seeking sometimes cannot be dissociated. For example, dopamine D1 receptor antagonist

$464 \mathrm{SCH} 23390$ attenuates overall locomotion and sucrose seeking, which are increased after 30

465 days of abstinence without extinction training (Grimm et al., 2011). Additionally, treatments that

466 reduce incubation of cocaine craving also reduce overall locomotion after $30+$ days of forced

467 abstinence (Murray et al., 2021). Thus, there is an overlapping system that contributes to

468 behavioral sensitization with drugs on board, cue-induced relapse-like behavior, and

469 locomotion. Here, we show that these drug-induced neural adaptations can persist and be

470 triggered by cue re-exposure even after prolonged abstinence.

471 In both sexes, chronic heroin exposure and extended abstinence results in an opioidspecific behavioral profile

475 of sex and reinforcer, other behaviors, like grooming and hyperactivity change uniquely

476 following exposure to heroin versus sucrose. Most of the research on incubation of craving has

477 utilized responses on a previously reward-paired lever to assess craving, regardless of 
478

479

480

481

482

483

484

485

486

487

488

489

490

491

492

493

494

495

496

497

498

499

500

501

502

503

reinforcer. Here, we show that other behaviors, such as grooming and hyperactivity, may be better suited for quantifying opioid craving. One important limitation of this study is that certain self-administration parameters (session duration and continuous versus intermittent access) were inconsistent across reinforcers, which possibly contributes to the behavioral differences seen here. Though a mechanism for driving these behavioral changes is undetermined, these unique behavioral profiles are significant and relevant because they 1) underscore the wealth of data indicating that increased active lever presses occur in males and females upon cue reexposure after prolonged abstinence, while 2) adding more nuanced and robust measurements of opioid craving specifically. These data can help improve preclinical models of craving/relapse-like behavior while also serving as a powerful foundation for additional mechanistic exploration.

The decrease in grooming and increase in hyperactivity that are associated with peak heroin craving may represent a hyperactive or compulsive behavioral profile similar to compulsive drug seeking. Noradrenergic hyperactivity can lead to hyperarousal, trouble focusing, and overactivity (Yamamoto et al., 2014). Treatments that reduce noradrenergic outflow have been shown to reduce cue-induced opioid craving (Sinha et al., 2007). Thus, one interpretation is that cue-induced heroin craving in late abstinence is reflective of a hyperactive state, a preoccupation with drug seeking, and a decrease in self-directed behaviors, such as grooming. Grooming and hyperactivity are associated with cocaine- and opioid-induced behavioral sensitization (Becker et al., 2001; Zhan et al., 2015), though not many studies have examined them in the context of cue-induced craving without re-exposure to the drug itself. This study lays the groundwork for further exploration of potential mechanisms underlying the behavioral profile associated with opioid-paired cue re-exposure.

It is interesting that many of the behaviors - incubation of craving, increased locomotion, and the heroin-specific decrease in grooming, and increase in hyperactivity- were consistently altered in both sexes. The only recorded behavior that was differentially affected in males and 
females was the sucrose-specific increase in sniffing seen in females, but not males. Notably, though sucrose-exposed females respond more than sucrose-exposed males during early abstinence, there were no observed sex differences in heroin seeking at either time point. This is consistent with other reports that males and females experience similar increases in opioidseeking behavior after extended abstinence (Venniro et al., 2019). Compared to males, females show enhanced incentive sensitization to cocaine following intermittent self-administration (Kawa and Robinson, 2019b), as well as behavioral sensitization to experimenter-delivered amphetamine (Van Haaren and Meyer, 1991). In some cases, behaviors like grooming are uniquely affected in only females and unchanged in males when morphine is on board (Zhan et al., 2015). Here, the magnitude of change in stereotypies and locomotion are similar in males and females, with the exception of sniffing in sucrose-exposed females. A possible explanation is that males and females react differently when opioids are present, but that over time, the opioid- and cue-induced behavioral changes become more comparable across sexes. This would help explain why males and females show a similar increase in cue-induced opioid craving over 30 days of abstinence (Venniro et al., 2019). Overall, this is promising in terms of designing treatments administered throughout abstinence that are likely to be effective in both sexes at reducing craving and other behaviors associated with compulsive drug seeking.

\section{Extended abstinence from heroin is associated with an increase in $\mathbf{5 0 - k H z}$ ultrasonic vocalizations in males, but not females}

Another sex-specific change was reflected in $50-\mathrm{kHz}$ ultrasonic vocalizations (USVs).

USVs were recorded during seeking tests to determine whether there is an affective component of drug/sucrose seeking in early versus late abstinence, when craving is high and other heroininduced behavioral changes emerge. Audio recording of craving tests on Day 1 and Day 30 of abstinence revealed no change in 22-kHz USVs in either sex, and an increase in 50-kHz USVs in males, but not females. $22-\mathrm{kHz}$ and $50-\mathrm{kHz}$ USVs were unchanged in sucrose-exposed males and females. The lack of $22-\mathrm{kHz}$ USVs is inconsistent with previous reports that when a 
531 conditioned cue is no longer predictive of a reward, rats will emit 22-kHz calls (Coffey et al., 532 2013). Here, there were few $22-\mathrm{kHz}$ calls during any of the cue tests for either sex/reinforcer.

533 The lack of $22-\mathrm{kHz}$ USVs is consistent with the idea that somatic withdrawal symptoms are not 534 driving craving behavior. Although rats may experience withdrawal-like behavior in early 535 abstinence following chronic opioid self-administration (Gipson et al., 2020; Seaman Jr and 536 Collins, 2021), withdrawal symptoms subside within the first few days of abstinence (Welsch et 537 al., 2020). Yet, craving continues to intensify for several weeks. As such, withdrawal signs and 538 craving follow opposite trajectories and one is unlikely to affect the other. Interestingly, 539 protracted forced abstinence from cocaine (30 or 60 days) does not lead to a difference in $22-$ $540 \mathrm{kHz}$ nor $50-\mathrm{kHz}$ calls during a contextual reinstatement test compared to calls made during self541 administration (Barker et al., 2014), though in this study, USVs were not collected during an 542 early abstinence seeking test. Here, $50-\mathrm{kHz}$, but not 22-kHz USVs change across abstinence in a sex- and reinforcer-specific manner.

544 Preclinical studies reveal that there are relatively fewer sex differences associated with 545 opioid-related behaviors, including relapse (Nicolas et al., 2021). Here, males and females show 546 similar patterns of relapse-like behavior, and yet females do not display an increase in $50-\mathrm{kHz}$ 547 USVs during the late abstinence relapse test when craving is high. It is likely that males emit 50$548 \mathrm{kHz}$ USVs as an anticipatory behavior during re-exposure to opioid-paired cues. This is 549 consistent with studies demonstrating that males report elevated moods prior to and during 550 cocaine and alcohol relapse events (McKay et al., 1996; Rubin et al., 1996). Other studies also 551 interpret $50-\mathrm{kHz}$ USVs as positive-valence affective states in anticipation of social and drug 552 rewards (Barfield et al., 1979; Ma et al., 2010). In fact, cues predictive of electrical stimulation of 553 reward-related brain regions elicit increased 50-kHz calls in males (Burgdorf et al., 2000).

554 Conversely, women are more prone to anxiety-, stress-, and depression-driven cocaine relapse 555 (reviewed in McKay et al., 1996; Rubin et al., 1996; Becker and Koob, 2016). Women report a 556 greater increase in sadness and decrease in joy in response to opioid-paired imagery and 
557 paraphernalia compared to men (Yu et al., 2007). As with many observed sex differences,

558 further research is needed to delineate why other behavioral end points are similar at

559 abstinence Day 30, and yet there is a sex difference in $50-\mathrm{kHz}$ USVs. In other studies examining USVs, females are often not included or not compared to males directly. One possibility is that females are more prone to stress-induced relapse. Re-exposure to drug-paired cues in the absence of drug delivery is likely to evoke mild stress (Schultz et al., 1997), and stress-induced reinstatement does increase over time (Sinha et al., 2011). However, it can be argued that the cue test is not as stressful as the stimuli used to precipitate stress-induced seeking, such as a foot shock. Therefore it is likely not sufficient to induce $22-\mathrm{kHz}$ calls nor 50 $\mathrm{kHz}$ calls in females, which would be consistent with a positive affect-induced relapse event. To that point, if a stress-primed reinstatement model was used in place of the cue-induced model here, females may show a greater number of USVs as they are potentially more sensitive to stress-induced relapse.

In males, the increase in $50-\mathrm{kHz}$ positive-valence affective calls, increase in locomotion, and increase in hyperactivity that accompany increased drug seeking may reflect an overactive

572 or "manic"-like state. The simultaneous decrease in grooming may reflect a decrease in selfdirected behaviors that is generally consistent with a compulsive drug seeking profile. This should be interpreted with caution so as not to over-anthropomorphize these measures. The intention is simply to characterize the behavioral profiles in a way that may lead to further exploration of underlying mechanisms. For example, these changes are potentially caused by dopamine D1 receptor activation in the nucleus accumbens shell, given that dopamine D1 receptors are linked to initiating anticipatory 50-kHz calls (Simola and Brudzynski, 2018), and D1 receptor antagonism reduces these calls (Buck et al., 2014). Of course, the dopamine system is also extensively linked to drug reward (Wise and Bozarth, 1985), motivation to seek rewards (reviewed in Volkow et al., 2017), as well as pathological manic states when there is an excess of dopamine (Ashok et al., 2017). Moreover, methamphetamine-induced 50-kHz calls 
583

584

585

586

587

588

589

590

591

592

593

594

595

596

597

598

599

600

601

602

603

604

605

606

607

608 are attenuated by anti-mania drugs and have, in the past, been used to quantify mania-like behavior in methamphetamine-treated rats (Wöhr, 2021). Additional studies are needed to ascertain the exact underlying mechanisms that connect increased locomotion, hyperactivity, and $50-\mathrm{kHz}$ calls to decreased grooming and increased heroin seeking; however, their coexistence could represent a state of hyperarousal and compulsive drug seeking possibly mediated by the dopamine system.

Females take increasing amounts of heroin and sucrose, and experience incubation of craving regardless of estrous cycle phase

Overall, this study shows few sex differences in terms of opioid-related behaviors. It is possible that sex differences will emerge when females are tested in a specific phase of the estrous cycle, and this effect may be obscured given the large sample sizes and estrous cycle variability across samples. Though there are many published reports indicating that the female estrous cycle impacts stimulant- and nicotine-related behaviors (Lynch, 2008; Feltenstein et al., 2009; Lynch et al., 2019; Nicolas et al., 2019), the results are equivocal when it comes to opioids, and there is very little available data concerning natural rewards like sucrose. Here, the female rats were freely cycling and estrous cycle was tracked throughout self-administration, as well as on days that they were tested for craving. There were a similar and ample number of females in non-estrus versus estrus on any given cue test day. Females self-administered increasing amounts of heroin and sucrose over the ten days of self-administration, and there were no differences in consumption between female rats in estrus versus those in non-estrus. All female rats experienced an increase in heroin- and sucrose-seeking behavior over 30 days of protracted abstinence, and there was no difference in lever pressing between estrus and nonestrus females at Day 1 nor Day 30. Thus, the increase in heroin and sucrose seeking cannot be attributed to a specific phase of the estrous cycle, nor is any one phase of the estrous cycle overrepresented here. 
Some data indicate that female rats self-administer up to $70 \%$ less heroin during the proestrus phase of their cycle (Lacy et al., 2016), and that ovariectomized (OVX) females treated with estradiol take a greater number of heroin infusions compared to vehicle-treated OVX females (Roth et al., 2002). However, these studies were either quite short (i.e. four hours in the case of the Lacy study), or a lower dose of heroin was used (i.e. $0.0075 \mathrm{mg} / \mathrm{kg}$ as in the case of the Roth study). In freely cycling females treated with vehicle and permitted to selfadminister heroin over an extended-access 12-hour session, there was no effect of estrous cycle on heroin taking (Roth et al., 2002). Other studies that utilized four three-hour daily sessions and a range of doses report no differences in heroin taking nor progressive ratio breakpoint between OVX females, OVX females treated with estradiol, and males (Stewart et al., 1996; Sedki et al., 2015). Though results regarding the role of gonadal hormones on opioid self-administration are conflicting across published reports, results from this study are more consistent with other extended-access paradigms.

One recent study found that rats self-administered similar amounts of sucrose regardless of estrous cycle phase (Schmidt et al., 2021). In this article, rats were also trained on heroin self-administration prior to sucrose self-administration, and there was an observed decrease in heroin taking during proestrus. However, heroin self-administration and sucrose self-administration sessions were both two hours in duration, and a lower heroin dose of 0.0075 $\mathrm{mg} / \mathrm{kg}$ was used. Thus, the sucrose self-administration paradigm used was similar to the one used here, whereas the heroin self-administration paradigm was not. Ours is the first study that the authors are aware of to track estrous cycle phase alongside incubation of sucrose craving. It has been reported that there are no sex differences in sucrose seeking after $1,21,60,120$, and 200 days of abstinence (estrous cycle was not tracked in this study) (Madangopal et al., 2019). Sucrose-related findings are extremely limited. Yet, results so far seem to be consistent with the finding that sucrose self-administration is not dependent on fluctuations in gonadal hormones. 
634 Locomotion is the only behavior enhanced in estrus females after $\mathbf{3 0}$ days of abstinence 635 from heroin, whereas locomotion and hyperactivity are enhanced in estrus females after 63630 days of abstinence from sucrose

Locomotion, sniffing, grooming, and hyperactivity were also assessed in the context of the estrous cycle. Estrus females showed increased locomotion following extended abstinence from both reinforcers. Sniffing and grooming were affected similarly in estrus versus non-estrus females at both abstinence time points for both reinforcers. Interestingly, although hyperactivity did not change across protracted abstinence in sucrose-exposed females, it was higher in estrus females at Day 30. Hyperactivity increased across over time in heroin-exposed females, but was not affected by estrous cycle phase. As with data regarding the effects of the estrous cycle on incubation of craving, there was a sizeable and comparable number of estrus and nonestrus females in any given group. Thus, the fact that males and females showed largely similar behavioral profiles in early and late abstinence is unlikely to be attributable to females being tested in a specific estrous cycle phase.

The overall increase in locomotion seen in estrus females builds on previous results indicating a general increase in activity in female rats in estrus (Giles et al., 2010) by demonstrating that this occurs reliably even after treatment with either sucrose or heroin. Other studies examining stimulant-induced behavioral changes show that estrus females demonstrated reduced cocaine- and amphetamine-induced locomotion and increased amphetamine-induced stereotypies than those in non-estrus (Becker and Cha, 1989; QuiñonesJenab et al., 1999). This is opposite from what was observed here in response to cues only: estrus females show an increase in locomotion, and no differences in other stereotyped behaviors. Interestingly, freely cycling females show cocaine-induced locomotor sensitization in response to cocaine challenge that is not seen in OVX females; however, OVX females treated with estradiol showed enhanced stereotyped behaviors and OVX females treated with progesterone showed reduced stereotyped behaviors (Souza et al., 2014). In these 
662

663

664

665

666

667

668

669

670

671

672

673

674

675

676

677

678

679

680

681

682

683

684

685

686

687

688

that, not only is overall locomotion increased following extended abstinence, it appears to be further enhanced in estrus females. Moreover, the increase in hyperactivity associated with heroin exposure is not attributable to the female estrous cycle since only sucrose-exposed estrus females show enhanced hyperactivity.

\section{Conclusions}

Cue-induced incubation of craving occurred similarly across 30 days of extended abstinence, regardless of sex and reinforcer. This increase in active lever pressing was associated with an increase in locomotion. Analysis of more granular behaviors revealed a drugspecific behavioral profile. Specifically, heroin exposure and extended abstinence were associated with a decrease in grooming and an increase in hyperactivity in both males and females that did not generalize to sucrose-exposed rats. Whereas increased responding on a previously reward-paired lever is suitable for assessing craving broadly (as has been the standard in the field), grooming and hyperactivity should be considered in future quantifications of opioid craving specifically. Therein, these data help to refine preclinical models of opioid relapse-like behavior. Heroin craving was also associated with an increase in $50-\mathrm{kHz}$ USVs in males, but not females. This indicates that males may experience a greater degree of anticipation and/or positive-valence affective states during opioid relapse events. Analysis of the female estrous cycle revealed that heroin and sucrose self-administration increase across ten days and are not different between estrus and non-estrus females. Similarly, all female rats showed enhanced craving after protracted abstinence, and there were no differences between estrus and non-estrus females in neither early nor late abstinence. Examination of addictionrelated behaviors in the context of the estrous cycle revealed that female rats in estrus showed enhanced locomotion, regardless of whether they self-administered sucrose or heroin. Interestingly, although hyperactivity was one of the heroin-specific changes, it was also enhanced in estrus females, though only those that had self-administered sucrose. Taken together, these results are a promising foundation for identifying neural mechanisms and 
689 behavioral signals associated with opioid craving in males and females. Additionally, they

690 suggest that potential treatments may not need to be quite as sensitive to fluctuations in 691 gonadal hormones in order to be effective.

692 


\section{References}

Aoyama K, Barnes J, Grimm JW (2014) Incubation of saccharin craving and within-session changes in responding for a cue previously associated with saccharin. Appetite 72:114122.

Ashok AH, Marques TR, Jauhar S, Nour MM, Goodwin GM, Young AH, Howes OD (2017) The dopamine hypothesis of bipolar affective disorder: the state of the art and implications for treatment. Mol Psychiatry 22:666-679.

Barfield RJ, Auerbach P, Geyer LA, Mclntosh TK (1979) Ultrasonic vocalizations in rat sexualbehavior. Am Zool 19:469-480.

Barker DJ, Simmons SJ, West MO (2015) Ultrasonic Vocalizations as a Measure of Affect in Preclinical Models of Drug Abuse: A Review of Current Findings. Current neuropharmacology 13:193-210.

Barker DJ, Bercovicz D, Servilio LC, Simmons SJ, Ma S, Root DH, Pawlak AP, West MO (2014) Rat ultrasonic vocalizations demonstrate that the motivation to contextually reinstate cocaine-seeking behavior does not necessarily involve a hedonic response. Addiction biology 19:781-790.

Becker JB, Cha J-H (1989) Estrous cycle-dependent variation in amphetamine-induced behaviors and striatal dopamine release assessed with microdialysis. Behavioural brain research 35:117-125.

Becker JB, Koob GF (2016) Sex Differences in Animal Models: Focus on Addiction. Pharmacological reviews 68:242-263.

Becker JB, Molenda H, Hummer DL (2001) Gender differences in the behavioral responses to cocaine and amphetamine. Implications for mechanisms mediating gender differences in drug abuse. Ann N Y Acad Sci 937:172-187.

Berridge KC, Robinson TE (2016) Liking, wanting, and the incentive-sensitization theory of addiction. The American psychologist 71:670-679.

Buck CL, Vendruscolo LF, Koob GF, George O (2014) Dopamine D1 and $\mu$-opioid receptor antagonism blocks anticipatory $50 \mathrm{kHz}$ ultrasonic vocalizations induced by palatable food cues in Wistar rats. Psychopharmacology 231:929-937.

Burgdorf J, Knutson B, Panksepp J (2000) Anticipation of rewarding electrical brain stimulation evokes ultrasonic vocalization in rats. Behavioral neuroscience 114:320-327.

Calipari ES, Juarez B, Morel C, Walker DM, Cahill ME, Ribeiro E, Roman-Ortiz C, Ramakrishnan C, Deisseroth K, Han MH, Nestler EJ (2017) Dopaminergic dynamics underlying sex-specific cocaine reward. Nature communications 8:13877.

Carr CC, Ferrario CR, Robinson TE (2020) Intermittent access cocaine self-administration produces psychomotor sensitization: effects of withdrawal, sex and cross-sensitization. Psychopharmacology 237:1795-1812. 
Coffey KR, Barker DJ, Ma S, Root DH, Martinez L, Horvitz JC, West MO (2013) Effects of varying reinforcement probability on pavlovian approach behavior and ultrasonic vocalizations in rats. Behavioural brain research 237:256-262.

Feltenstein MW, Byrd EA, Henderson AR, See RE (2009) Attenuation of cocaine-seeking by progesterone treatment in female rats. Psychoneuroendocrinology 34:343-352.

Gawin FH, Kleber HD (1986) Abstinence symptomatology and psychiatric diagnosis in cocaine abusers. Clinical observations. Archives of general psychiatry 43:107-113.

Giles ED, Jackman MR, Johnson GC, Schedin PJ, Houser JL, MacLean PS (2010) Effect of the estrous cycle and surgical ovariectomy on energy balance, fuel utilization, and physical activity in lean and obese female rats. American journal of physiology Regulatory, integrative and comparative physiology 299:R1634-1642.

Gipson CD, Dunn KE, Bull A, Ulangkaya H, Hossain A (2020) Establishing preclinical withdrawal syndrome symptomatology following heroin self-administration in male and female rats. Experimental and clinical psychopharmacology.

Grimm JW, Hope BT, Wise RA, Shaham Y (2001) Neuroadaptation. Incubation of cocaine craving after withdrawal. Nature 412:141-142.

Grimm JW, Manaois M, Osincup D, Wells B, Buse C (2007) Naloxone attenuates incubated sucrose craving in rats. Psychopharmacology 194:537-544.

Grimm JW, Harkness JH, Ratliff C, Barnes J, North K, Collins S (2011) Effects of systemic or nucleus accumbens-directed dopamine D1 receptor antagonism on sucrose seeking in rats. Psychopharmacology 216:219-233.

Harkness JH, Wells J, Webb S, Grimm JW (2016) Extended exposure to environmental cues, but not to sucrose, reduces sucrose cue reactivity in rats. Learning \& Behavior 44:59-66.

Kawa AB, Robinson TE (2019a) Sex differences in incentive-sensitization produced by intermittent access cocaine self-administration. Psychopharmacology 236:625-639.

Kawa AB, Robinson TE (2019b) Sex differences in incentive-sensitization produced by intermittent access cocaine self-administration. Psychopharmacology 236:625-639.

Lacy RT, Strickland JC, Feinstein MA, Robinson AM, Smith MA (2016) The effects of sex, estrous cycle, and social contact on cocaine and heroin self-administration in rats. Psychopharmacology 233:3201-3210.

Leri F, Flores J, Rajabi H, Stewart J (2003) Effects of Cocaine in Rats Exposed to Heroin. Neuropsychopharmacology : official publication of the American College of Neuropsychopharmacology 28:2102-2116.

Lu L, Grimm JW, Hope BT, Shaham Y (2004) Incubation of cocaine craving after withdrawal: a review of preclinical data. Neuropharmacology 47 Suppl 1:214-226.

Lynch WJ (2008) Acquisition and maintenance of cocaine self-administration in adolescent rats: effects of sex and gonadal hormones. Psychopharmacology 197:237-246. 
Lynch WJ, Tan L, Narmeen S, Beiter R, Brunzell DH (2019) Exercise or saccharin during abstinence block estrus-induced increases in nicotine-seeking. Physiology \& behavior 203:33-41.

Ma ST, Maier EY, Ahrens AM, Schallert T, Duvauchelle CL (2010) Repeated intravenous cocaine experience: development and escalation of pre-drug anticipatory $50-\mathrm{kHz}$ ultrasonic vocalizations in rats. Behavioural brain research 212:109-114.

Madangopal R, Tunstall BJ, Komer LE, Weber SJ, Hoots JK, Lennon VA, Bossert JM, Epstein DH, Shaham Y, Hope BT (2019) Discriminative stimuli are sufficient for incubation of cocaine craving. eLife 8.

McKay JR, Rutherford MJ, Cacciola JS, Kabasakalian-McKay R, Alterman AI (1996) Gender differences in the relapse experiences of cocaine patients. The Journal of nervous and mental disease 184:616-622.

Mittal N, Thakore N, Bell RL, Maddox WT, Schallert T, Duvauchelle CL (2019) Sex-specific ultrasonic vocalization patterns and alcohol consumption in high alcohol-drinking (HAD1) rats. Physiology \& behavior 203:81-90.

Murray $\mathrm{CH}$, Christian DT, Milovanovic M, Loweth JA, Hwang EK, Caccamise AJ, Funke JR, Wolf ME (2021) mGlu5 function in the nucleus accumbens core during the incubation of methamphetamine craving. Neuropharmacology 186:108452.

Nicolas C, Zlebnik NE, Farokhnia M, Leggio L, Ikemoto S, Shaham Y (2021) Sex differences in opioid and psychostimulant craving and relapse: a critical review. medRxiv:2021.2003.2030.21254644.

Nicolas C, Russell TI, Pierce AF, Maldera S, Holley A, You ZB, McCarthy MM, Shaham Y, Ikemoto $S$ (2019) Incubation of Cocaine Craving After Intermittent-Access Selfadministration: Sex Differences and Estrous Cycle. Biological psychiatry 85:915-924.

O'Brien CP, Childress AR, McLellan AT, Ehrman R (1992) Classical Conditioning in DrugDependent Humansa. Annals of the New York Academy of Sciences 654:400-415.

Paris JJ, Fenwick J, McLaughlin JP (2014) Estrous cycle and HIV-1 Tat protein influence cocaine-conditioned place preference and induced locomotion of female mice. Current HIV research 12:388-396.

Paulson PE, Camp DM, Robinson TE (1991) Time course of transient behavioral depression and persistent behavioral sensitization in relation to regional brain monoamine concentrations during amphetamine withdrawal in rats. Psychopharmacology 103:480492.

Pickens CL, Airavaara M, Theberge F, Fanous S, Hope BT, Shaham Y (2011) Neurobiology of the incubation of drug craving. Trends in neurosciences 34:411-420.

Powell KR, Holtzman SG (2001) Parametric evaluation of the development of sensitization to the effects of morphine on locomotor activity. Drug and alcohol dependence 62:83-90. 
Quiñones-Jenab V, Ho A, Schlussman SD, Franck J, Kreek MJ (1999) Estrous cycle differences in cocaine-induced stereotypic and locomotor behaviors in Fischer rats. Behavioural brain research 101:15-20.

Robinson TE (1984) Behavioral sensitization: characterization of enduring changes in rotational behavior produced by intermittent injections of amphetamine in male and female rats. Psychopharmacology 84:466-475.

Roth ME, Casimir AG, Carroll ME (2002) Influence of estrogen in the acquisition of intravenously self-administered heroin in female rats. Pharmacology, biochemistry, and behavior 72:313-318.

Rubin A, Stout RL, Longabaugh R (1996) Gender differences in relapse situations. Addiction (Abingdon, England) 91 Suppl:S111-120.

Sax KW, Strakowski SM (2001) Behavioral sensitization in humans. Journal of addictive diseases 20:55-65.

Schmidt KT, Sharp JL, Ethridge SB, Pearson T, Ballard S, Potter KM, Smith MA (2021) The effects of strain and estrous cycle on heroin- and sugar-maintained responding in female rats. Behavioural brain research 409:113329.

Schultz W, Dayan P, Montague PR (1997) A neural substrate of prediction and reward. Science (New York, NY) 275:1593-1599.

Seaman Jr RW, Collins GT (2021) Impact of Morphine Dependence and Withdrawal on the Reinforcing Effectiveness of Fentanyl, Cocaine, and Methamphetamine in Rats. Front Pharmacol 12.

Sedki F, Gardner Gregory J, Luminare A, D'Cunha TM, Shalev U (2015) Food restrictioninduced augmentation of heroin seeking in female rats: manipulations of ovarian hormones. Psychopharmacology 232:3773-3782.

Simola N, Brudzynski S (2018) Rat 50-kHz ultrasonic vocalizations as a tool in studying neurochemical mechanisms that regulate positive emotional states. Journal of Neuroscience Methods 310.

Sinha R, Shaham Y, Heilig M (2011) Translational and reverse translational research on the role of stress in drug craving and relapse. Psychopharmacology 218:69-82.

Sinha R, Kimmerling A, Doebrick C, Kosten TR (2007) Effects of lofexidine on stress-induced and cue-induced opioid craving and opioid abstinence rates: preliminary findings. Psychopharmacology 190:569-574.

Souza MF, Couto-Pereira NS, Freese L, Costa PA, Caletti G, Bisognin KM, Nin MS, Gomez R, Barros HM (2014) Behavioral effects of endogenous or exogenous estradiol and progesterone on cocaine sensitization in female rats. Brazilian journal of medical and biological research $=$ Revista brasileira de pesquisas medicas e biologicas 47:505-514.

Steketee JD, Kalivas PW (2011) Drug wanting: behavioral sensitization and relapse to drugseeking behavior. Pharmacological reviews 63:348-365. 
Stewart J, Woodside B, Shaham Y (1996) Ovarian hormones do not affect the initiation and maintenance of intravenous self-administration of heroin in the female rat. Psychobiology 24:154-159.

Tindell AJ, Berridge KC, Zhang J, Peciña S, Aldridge JW (2005) Ventral pallidal neurons code incentive motivation: amplification by mesolimbic sensitization and amphetamine. The European journal of neuroscience 22:2617-2634.

Van Haaren F, Meyer ME (1991) Sex differences in locomotor activity after acute and chronic cocaine administration. Pharmacology Biochemistry and Behavior 39:923-927.

Vanderschuren LJ, Tjon GH, Nestby P, Mulder AH, Schoffelmeer AN, De Vries TJ (1997) Morphine-induced long-term sensitization to the locomotor effects of morphine and amphetamine depends on the temporal pattern of the pretreatment regimen. Psychopharmacology 131:115-122.

Venniro M, Russell TI, Zhang M, Shaham Y (2019) Operant Social Reward Decreases Incubation of Heroin Craving in Male and Female Rats. Biological psychiatry 86:848856.

Volkow ND, Wise RA, Baler R (2017) The dopamine motive system: implications for drug and food addiction. Nature reviews Neuroscience 18:741-752.

Welsch L, Bailly J, Darcq E, Kieffer BL (2020) The Negative Affect of Protracted Opioid Abstinence: Progress and Perspectives From Rodent Models. Biological psychiatry 87:54-63.

Wise RA (2004) Dopamine, learning and motivation. Nature Reviews Neuroscience 5:483-494.

Wise RA, Bozarth MA (1985) Brain mechanisms of drug reward and euphoria. Psychiatr Med 3:445-460.

Witkiewitz K, Marlatt GA (2004) Relapse prevention for alcohol and drug problems: that was Zen, this is Tao. The American psychologist 59:224-235.

Witkiewitz K, Bowen S (2010) Depression, craving, and substance use following a randomized trial of mindfulness-based relapse prevention. J Consult Clin Psychol 78:362-374.

Wöhr M (2021) Measuring mania-like elevated mood through amphetamine-induced 50-kHz ultrasonic vocalizations in rats. British Journal of Pharmacology n/a.

Wu M, Brudzynski SM, Mogenson GJ (1993) Functional interaction of dopamine and glutamate in the nucleus accumbens in the regulation of locomotion. Canadian journal of physiology and pharmacology 71:407-413.

Yamamoto K, Shinba T, Yoshii M (2014) Psychiatric symptoms of noradrenergic dysfunction: a pathophysiological view. Psychiatry and clinical neurosciences 68:1-20.

Yu J, Zhang S, Epstein DH, Fang Y, Shi J, Qin H, Yao S, Le Foll B, Lu L (2007) Gender and stimulus difference in cue-induced responses in abstinent heroin users. Pharmacology Biochemistry and Behavior 86:485-492. 
Zhan B, Ma HY, Wang JL, Liu CB (2015) Sex differences in morphine-induced behavioral sensitization and social behaviors in ICR mice. Dong wu xue yan jiu = Zoological

881

882 research 36:103-108. 
Figure 1. Incubation of craving is associated with sex- and reinforcer-specific behavioral signatures. A. Experimental timeline including 10 days of intravenous heroin or oral sucrose self-administration, followed by a recorded cue test after one or 30 days of forced abstinence. B. Cameras were mounted on the ceiling of the sound-attenuating chamber to record movement and behaviors during the cue test. An ultrasonic microphone was positioned flush against the Plexiglas top of the operant box to record ultrasonic vocalizations. C. Sucrose- and heroinexposed males and females showed incubation of craving, as indicated by an increase in active levers after 30 days of abstinence compared to one day of abstinence. Post-hoc comparisons revealed that sucrose females responded significantly more than heroin females and sucrose males in early abstinence (day $\left[F_{1,232}=52.74, p<.0001\right]$; Day 1 female sucrose vs Day 1 female heroin, $p=.0003$; Day 1 female sucrose vs Day 1 male sucrose, $p=.0402$, Bonferroni's multiple comparisons test). D. Overall locomotion, as measured by total beam breaks increased in heroin- and sucrose-exposed males and females over 30 days of abstinence (day $\left[F_{1,232}=100.6\right.$, $p<.0001])$. E. Grooming decreased in heroin-exposed rats and was unchanged in sucroseexposed rats of both sexes after 30 days of abstinence (interaction $\left[F_{1,232}=4.318, p=.0388\right]$; Day 1 male heroin vs Day 30 male heroin, $p=.0138$; Day 1 male sucrose vs Day 30 male sucrose, $p>$.9999; Day 1 female heroin vs Day 30 female heroin, $p<.0001$; Day 1 female sucrose vs Day 30 female sucrose, $p=.1848$, Bonferroni's multiple comparisons test). F. Sniffing was unchanged in all conditions, with the exception of an increase in sucrose-exposed females. Post-hoc comparisons revealed that sucrose males engaged in more sniffing than heroin males in late abstinence, and more sniffing than sucrose females in early abstinence (interaction $\left[F_{1,232}=4.304, p=.0391\right]$; Day 1 male heroin vs Day 30 male heroin, $p>.9999$; Day 1 male sucrose vs Day 30 male sucrose, $p=.1316$; Day 1 female heroin vs Day 30 female heroin; Day 1 female sucrose vs Day 30 female sucrose, $p<.0001, p=.1848$, Bonferroni's multiple comparisons test). G. Hyperactivity, as measured by hops and darts across the cage, increased over 30 days of abstinence from heroin, but not sucrose in males and females (interaction $\left[F_{1,232}=12.11\right.$, $p=.0006]$; Day 1 male heroin vs Day 30 male heroin, $p=.0050$; Day 1 male sucrose vs Day 30 male sucrose, $p>$.9999; Day 1 female heroin vs Day 30 female heroin, $p<.0001$; Day 1 female sucrose vs Day 30 female sucrose, $p=.7376$, Bonferroni's multiple comparisons test). Additional statistics are listed in Table 1. Data show mean \pm SEM; ${ }^{*} p<.05$.

Figure 2. Incubation of heroin craving is associated with an increase in $50-\mathrm{kHz}$ ultrasonic vocalizations (USVs) in males only (interaction $\left[F_{1,51}=60.12, p<.0001\right]$; Day 1 heroin male 50$\mathrm{kHz}$ calls vs Day 30 heroin male $50-\mathrm{kHz}$ calls, $p<.0001$; Day 1 sucrose male $50-\mathrm{kHz}$ calls vs Day 30 sucrose male 50-kHz calls, $p>$.9999; Day 1 heroin female $50-k H z$ calls vs Day 30 heroin female $50-\mathrm{kHz}$ calls, $p>$.9999; Day 1 sucrose female $50-\mathrm{kHz}$ calls vs Day 30 sucrose female 50 $\mathrm{kHz}$ calls, $p>$.9999, Bonferroni's multiple comparisons test). Additional statistics are listed in Table 1. Data show mean $\pm \mathrm{SEM} ;{ }^{*} \mathrm{p}<.05$.

Table 1. Additional statistics associated with results from Figure 1 and Figure 2.

Figure 3. The effect of the female estrous cycle phase (estrus versus non-estrus) on heroin and sucrose self-administration and craving. A. Female heroin-treated rats earned more infusions over 10 days of intravenous self-administration regardless of estrous cycle phase (time, $\left[F_{9,541}=3.821, p=.0001\right]$, estrus, $\left[F_{3,148}=.5299, p=.6624\right]$; interaction, $\left.\left[F_{27,541}=4.405, p<.0001\right]\right)$. B. Females demonstrated incubation of heroin craving, as indicated by an increase in active lever responses during a 30-minute cue test following 30 days of abstinence, and there were no differences in active lever responses between estrus and non-estrus females at either abstinence time point (time, $\left[F_{1,51}=18.02, p<.0001\right]$, estrus, $\left[F_{1,51}=.0881, p=.7677\right]$; interaction, $\left.\left[F_{1,51}=1.989, p=.1645\right]\right)$. C. Female sucrose-treated rats earned more sucrose pellets over 10 
days of oral self-administration regardless of estrous cycle phase (time, $\left[F_{9,760}=66.68, p<.0001\right]$, estrus, $\left[F_{1,201}=.2342, p=.6290\right]$; interaction, $\left.\left[F_{9,760}=.8406, p=.5787\right]\right)$. D. Females demonstrated incubation of sucrose craving, as indicated by an increase in active lever responses during a 1hour cue test following 30 days of abstinence, and there were no differences in active lever responses between estrus and non-estrus females at either abstinence time point (time, [ $\left.F_{1,63}=12.75, p=.0007\right]$, estrus, [ $\left.F_{1,63}=.2057, p=.6517\right]$; interaction, [ $\left.\left.F_{1,63}=.7248, p=.6517\right]\right)$. Data show mean $\pm \mathrm{SEM} ;{ }^{*} \mathrm{p}<.05$.

Figure 4. The effect of the female estrous cycle phase (estrus versus non-estrus) on addictionrelated behaviors following one or 30 days of abstinence from heroin. A. Total beam breaks increased over 30 days of abstinence, and were enhanced in estrus females compared to nonestrus females (time $\left[F_{1,45}=33.58, p<.0001\right]$; estrus, $\left[F_{1,45}=6.151, p=.0169\right]$; interaction, $\left.\left[F_{1,45}=0.5535, p=.4608\right]\right)$. B. Grooming decreased over 30 days of abstinence regardless of estrous cycle phase (time, $\left[F_{1,45}=18.30, p=.0001\right]$; estrus, $\left[F_{1,45}=0.0093, p=.9235\right]$; interaction, $\left.\left[F_{1,45}=1.762, p=.1911\right]\right)$. C. Sniffing increased over 30 days of abstinence regardless of estrous cycle phase (time, $\left[F_{1,45}=9.245, p=.0039\right]$; estrus, $\left[F_{1,45}=1.018, p=.3183\right]$; interaction, $\left.\left[F_{1,45}=0.0221, p=.8826\right]\right)$. D. Hyperactivity increased over 30 days of abstinence and there was no effect of estrous cycle phase (time, [ $\left.F_{1,45}=15.36, p=.0003\right]$; estrus, [ $\left.F_{1,45}=0.0558, p=.8144\right]$; interaction, $\left.\left[F_{1,45}=0.2112, p=.6481\right]\right)$. Data show mean $\pm \mathrm{SEM} ;{ }^{*} \mathrm{p}<.05$.

Figure 5. The effect of the female estrous cycle phase (estrus versus non-estrus) on addictionrelated behaviors following one or 30 days of abstinence from sucrose. A. Total beam breaks increased over 30 days of abstinence, and were enhanced in estrus females compared to nonestrus females (time, [ $\left.F_{1,86}=54.91, p<.0001\right]$; estrus, $\left[F_{1,86}=6.386, p=.0133\right]$; interaction, $\left.\left[F_{1,86}=0.0115, p=.9150\right]\right)$. B. Grooming increased over 30 days of abstinence regardless of estrous cycle phase (time [ $\left.F_{1,86}=4.194, p=.0436\right]$ ); estrus, $\left[F_{1,86}=1.091, p=.2993\right]$; interaction, $\left.\left[F_{1,86}=1.974, p=.11636\right]\right)$. C. Sniffing increased over 30 days of abstinence regardless of estrous cycle phase (time $\left[F_{1,86}=23.98, p<0.0001\right.$; estrus, $\left[F_{1,86}=0.003, p=.9568\right]$; interaction, $\left.\left[F_{1,86}=0.064, p=.8006\right]\right)$. D. Hyperactivity was enhanced at Day 30 in estrus females, and there was no effect of estrous cycle on hyperactivity at Day 1 (time, $\left[F_{1,86}=3.923, p=.0508\right]$; estrus, $\left[F_{1,86}=5.459, p=.0218\right]$; interaction, $\left[F_{1,86}=1.767, p=.1872\right]$; Day 1 estrus vs non-estrus, $p>0.9999$; Day 30 estrus vs non-estrus, $p=0.0479$, Bonferroni's multiple comparisons test). Data show mean $\pm \mathrm{SEM} ;{ }^{*} \mathrm{p}<.05$. 
A

Sucrose

or Heroin

Self-Administration

Abstinence

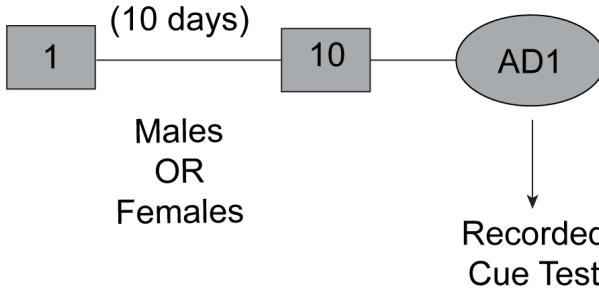

B
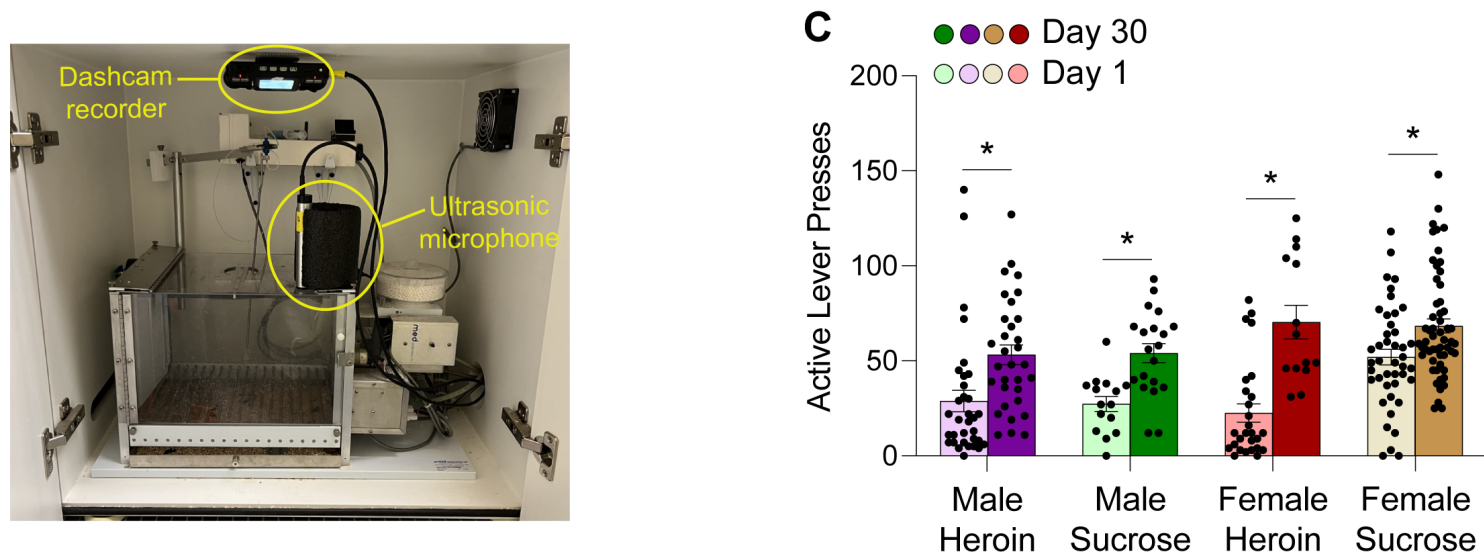

D

E
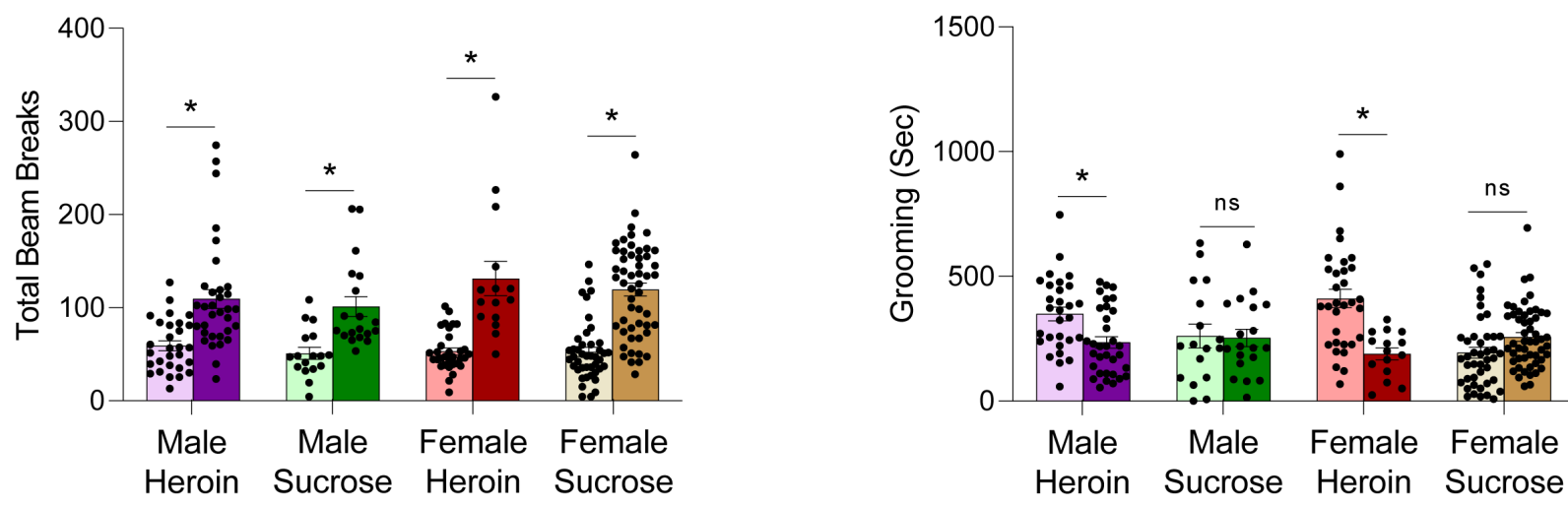

F

G
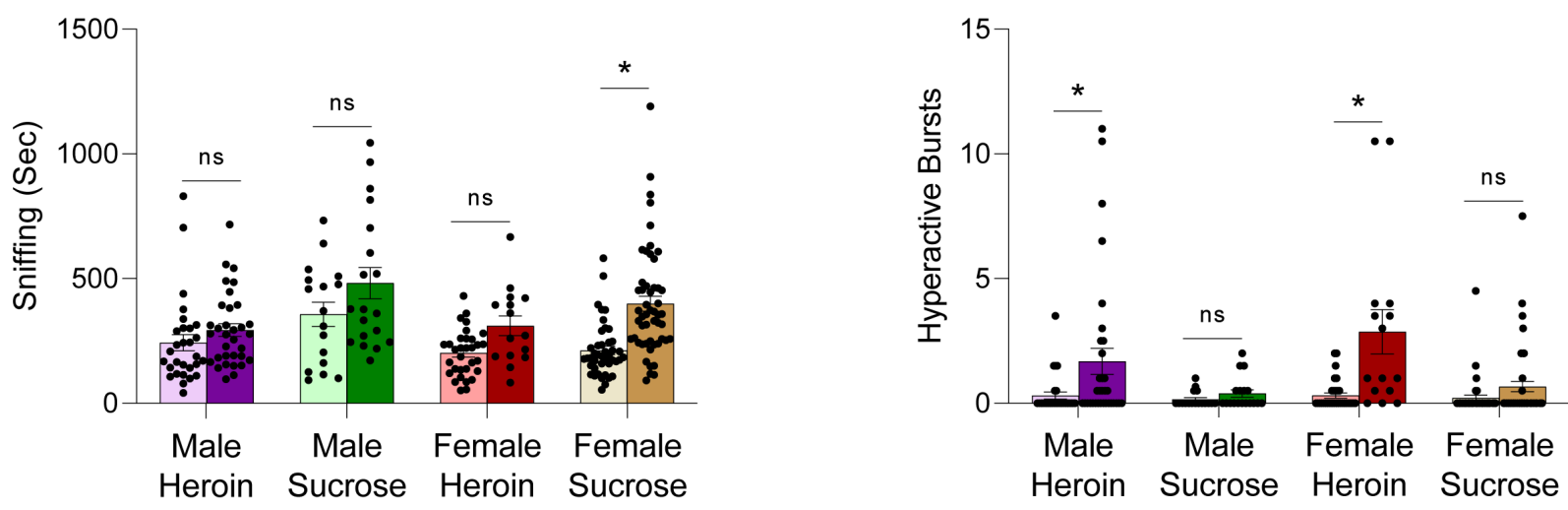


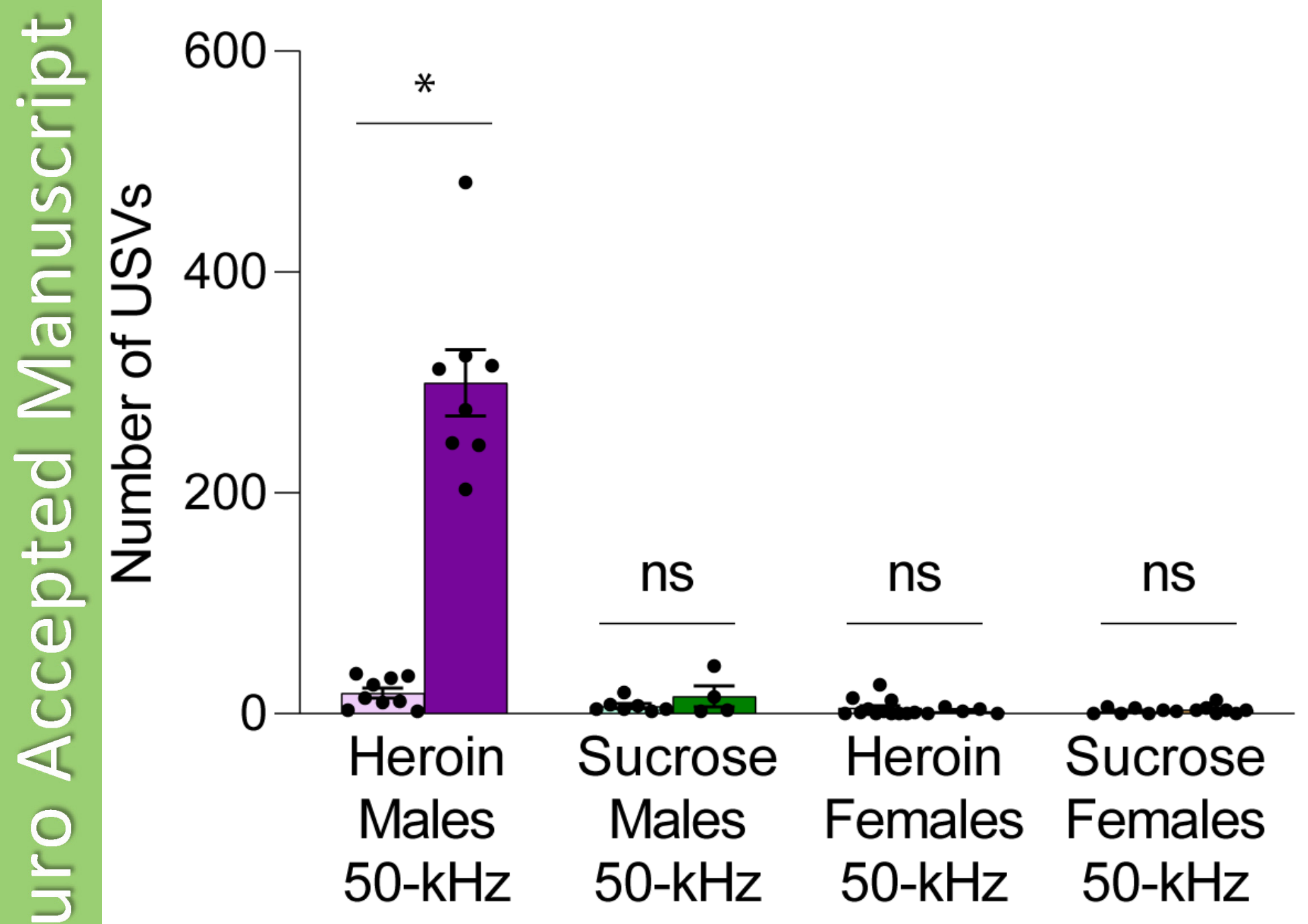


는

$\frac{n}{\sqrt{2}}$

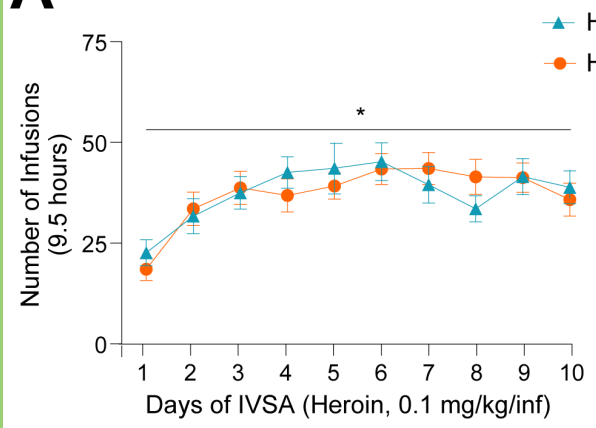

(0)

(d) $\mathrm{C}$

+1)

(d)

(u)

(c)

$<$

(2)

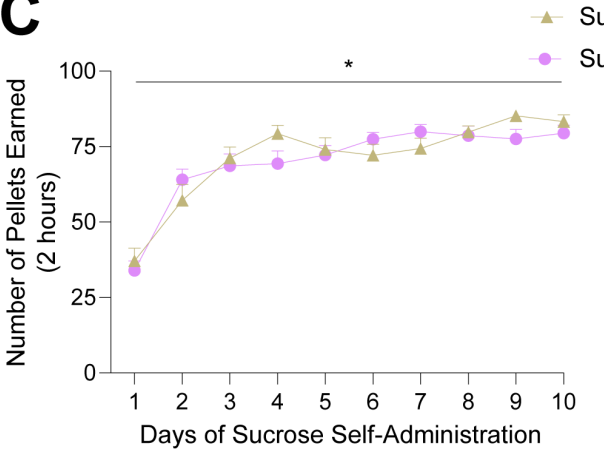

B

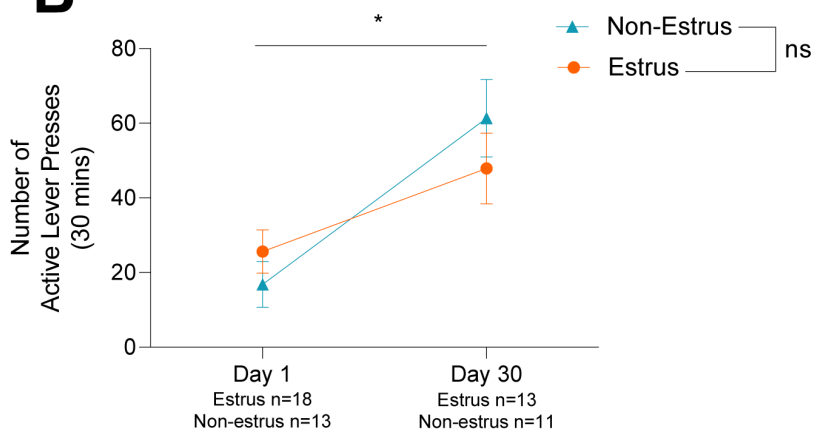

D

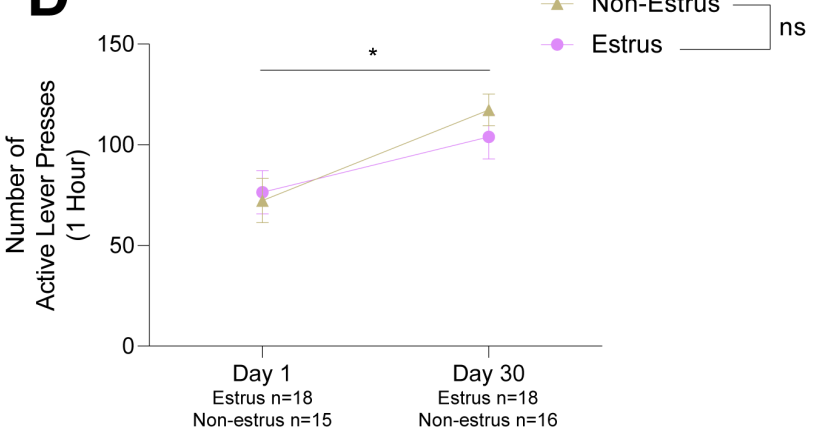


A Heroin Total Beam Breaks $\quad$ B

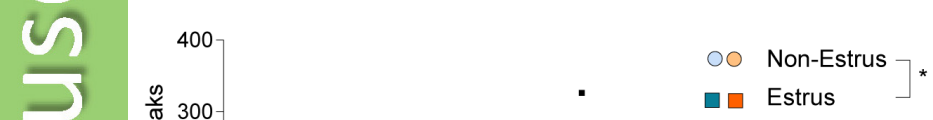

घ- Estrus

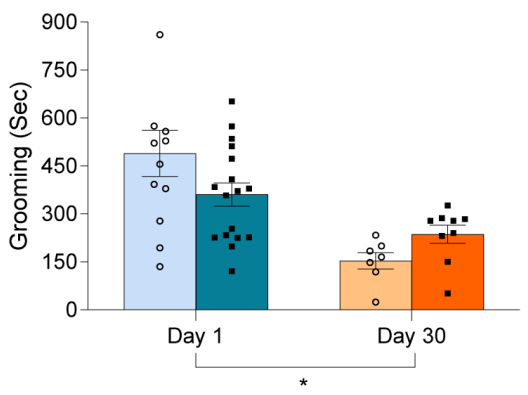

$\left.\begin{array}{ll}0 & \text { Non-Estrus } \\ \square & \text { Estrus }\end{array}\right] \mathrm{ns}$

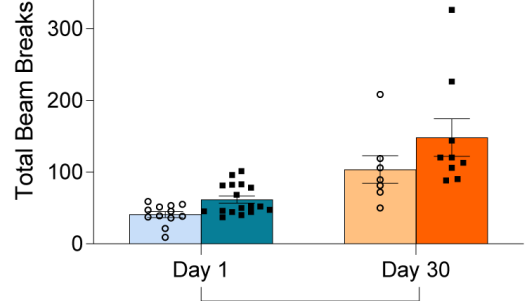

D

Heroin Sniffing

Heroin Hyperactivity

11

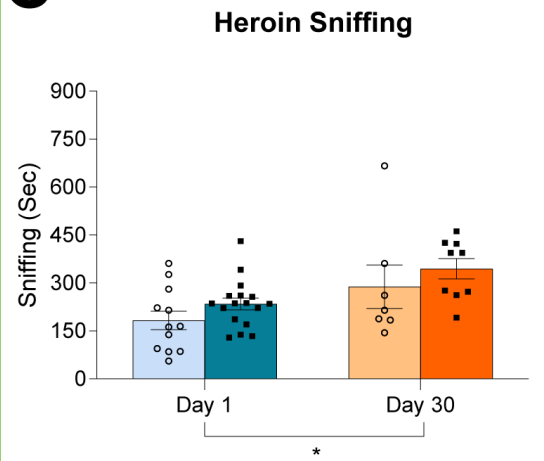

O० Non-Estrus $] \mathrm{ns}$

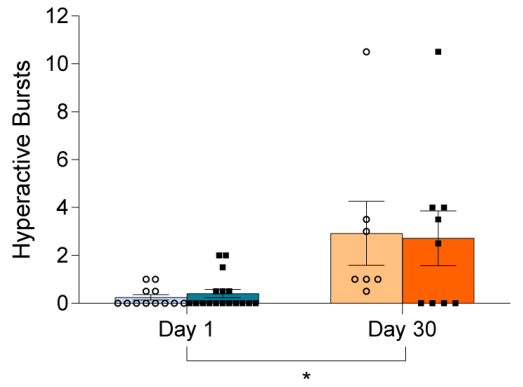


Sucrose Total Beam Breaks
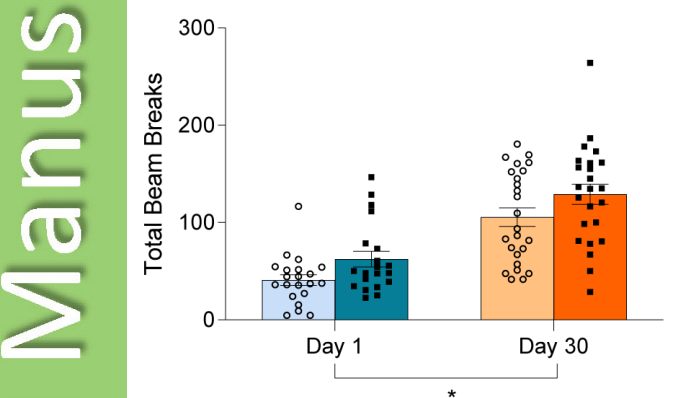

Sucrose Sniffing

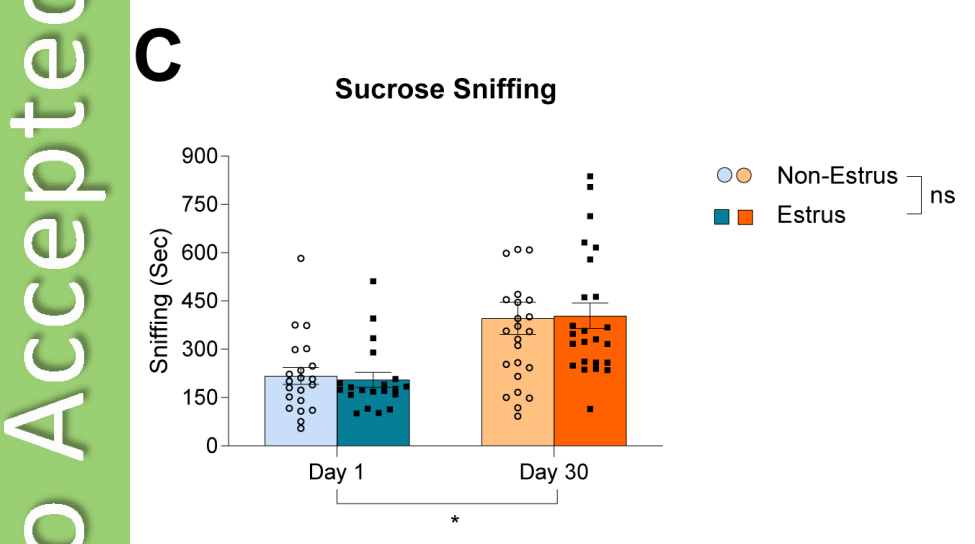

Non-Estrus $]$ Estrus
B

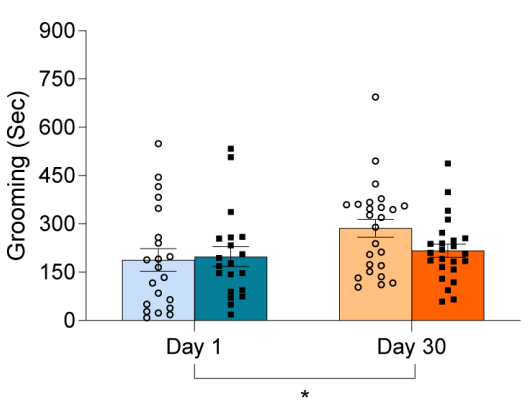

0 Non-Estrus $]$ ns

D

Sucrose Hyperactivity

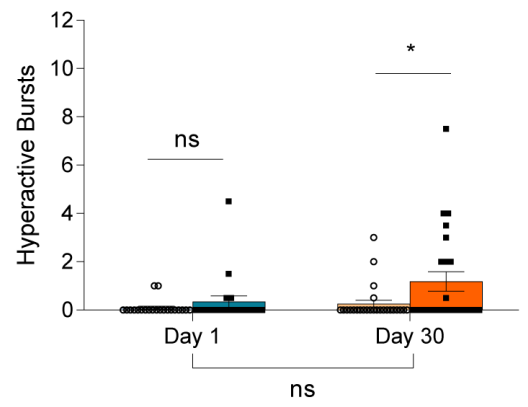

$\left.\begin{array}{l}\text { Non-Estrus } \\ \text { Estrus }\end{array}\right]$ * 


\begin{tabular}{|c|c|c|c|}
\hline Figure & Factors & F values & p-values \\
\hline \multirow{7}{*}{ Figure 1C } & Day & $F(1,232)=52.74$ & $\mathrm{P}<0.0001$ \\
\hline & Sex & $F(1,232)=9.825$ & $\mathrm{P}=0.0019$ \\
\hline & Reinforcer & $F(1,232)=2.824$ & $\mathrm{P}=0.0942$ \\
\hline & Day x Sex & $F(1,232)=0.6877$ & $\mathrm{P}=0.4078$ \\
\hline & Day x Reinforcer & $F(1,232)=3.340$ & $\mathrm{P}=0.0689$ \\
\hline & Sex x Reinforcer & $\mathrm{F}(1,232)=3.139$ & $\mathrm{P}=0.0777$ \\
\hline & Day x Sex x Reinforcer & $\mathrm{F}(1,232)=4.549$ & $\mathrm{P}=0.0340$ \\
\hline \multirow{7}{*}{ Figure 1D } & Day & $\mathrm{F}(1,232)=100.6$ & $\mathrm{P}<0.0001$ \\
\hline & Sex & $\mathrm{F}(1,232)=2.124$ & $\mathrm{P}=0.1464$ \\
\hline & Reinforcer & $\mathrm{F}(1,232)=1.397$ & $\mathrm{P}=0.2384$ \\
\hline & Day x Sex & $F(1,232)=3.380$ & $\mathrm{P}=0.0673$ \\
\hline & Day x Reinforcer & $F(1,232)=0.2113$ & $\mathrm{P}=0.6461$ \\
\hline & Sex x Reinforcer & $\mathrm{F}(1,232)=0.02831$ & $\mathrm{P}=0.8665$ \\
\hline & Day x Sex x Reinforcer & $F(1,232)=0.2019$ & $\mathrm{P}=0.6536$ \\
\hline \multirow{7}{*}{ Figure 1E } & Day & $\mathrm{F}(1,232)=10.78$ & $\mathrm{P}=0.0012$ \\
\hline & Sex & $F(1,232)=0.2969$ & $\mathrm{P}=0.5864$ \\
\hline & Reinforcer & $F(1,232)=6.620$ & $\mathrm{P}=0.0107$ \\
\hline & Day x Sex & $F(1,232)=0.1863$ & $\mathrm{P}=0.6664$ \\
\hline & Day x Reinforcer & $F(1,232)=20.91$ & $\mathrm{P}<0.0001$ \\
\hline & Sex x Reinforcer & $F(1,232)=0.8497$ & $\mathrm{P}=0.3576$ \\
\hline & Day x Sex x Reinforcer & $\mathrm{F}(1,232)=4.318$ & $\mathrm{P}=0.0388$ \\
\hline \multirow{7}{*}{ Figure $1 \mathrm{~F}$} & Day & $\mathrm{F}(1,232)=23.20$ & $\mathrm{P}<0.0001$ \\
\hline & Sex & $F(1,232)=6.569$ & $\mathrm{P}=0.0110$ \\
\hline & Reinforcer & $F(1,232)=16.82$ & $\mathrm{P}<0.0001$ \\
\hline & Day x Sex & $\mathrm{F}(1,232)=1.523$ & $\mathrm{P}=0.2184$ \\
\hline & Day x Reinforcer & $F(1,232)=2.408$ & $\mathrm{P}=0.1221$ \\
\hline & Sex x Reinforcer & $F(1,232)=4.304$ & $\mathrm{P}=0.0391$ \\
\hline & Day x Sex x Reinforcer & $\mathrm{F}(1,232)=0.001682$ & $\mathrm{P}=0.9673$ \\
\hline \multirow{7}{*}{ Figure $1 \mathrm{G}$} & Day & $\mathrm{F}(1,231)=24.49$ & $\mathrm{P}<0.0001$ \\
\hline & Sex & $\mathrm{F}(1,231)=2.717$ & $\mathrm{P}=0.1007$ \\
\hline & Reinforcer & $F(1,231)=16.05$ & $\mathrm{P}<0.0001$ \\
\hline & Day x Sex & $F(1,231)=2.268$ & $\mathrm{P}=0.1334$ \\
\hline & Day x Reinforcer & $\mathrm{F}(1,231)=12.11$ & $\mathrm{P}=0.0006$ \\
\hline & Sex x Reinforcer & $F(1,231)=0.8109$ & $\mathrm{P}=0.3688$ \\
\hline & Day x Sex x Reinforcer & $F(1,231)=1.068$ & $\mathrm{P}=0.3025$ \\
\hline \multirow{7}{*}{ Figure 2} & Day & $F(1,51)=65.73$ & $\mathrm{P}<0.0001$ \\
\hline & Sex & $F(1,51)=84.83$ & $\mathrm{P}<0.0001$ \\
\hline & Reinforcer & $F(1,51)=69.82$ & $\mathrm{P}<0.0001$ \\
\hline & Day x Sex & $F(1,51)=67.04$ & $\mathrm{P}<0.0001$ \\
\hline & Day x Reinforcer & $F(1,51)=56.88$ & $\mathrm{P}<0.0001$ \\
\hline & Sex x Reinforcer & $F(1,51)=68.35$ & $\mathrm{P}<0.0001$ \\
\hline & Day x Sex x Reinforcer & $F(1,51)=60.12$ & $\mathrm{P}<0.0001$ \\
\hline
\end{tabular}

